\title{
Tentonin 3/TMEM150C senses blood pressure changes in the aortic arch
}

\author{
Huan-Jun Lu, ${ }^{1,2}$ Thien-Luan Nguyen, ${ }^{1,2}$ Gyu-Sang Hong, ${ }^{1}$ Sungmin Pak, ${ }^{1}$ Hyesu Kim, ${ }^{1}$ Hyungsup Kim, ${ }^{1}$ Dong-Yoon Kim, ${ }^{3}$ \\ Sung-Yon Kim, ${ }^{3}$ Yiming Shen, ${ }^{4}$ Pan Dong Ryu, ${ }^{4}$ Mi-Ock Lee, ${ }^{2}$ and Uhtaek $\mathrm{Oh}^{1}$ \\ BBrain Science Institute, Korea Institute of Science and Technology (KIST), Seoul, Korea. ${ }^{2}$ College of Pharmacy, Institute of Molecular Biology and Genetics, and ${ }^{4}$ College of Veterinary Medicine, Seoul \\ National University, Seoul, Korea.
}

\begin{abstract}
The baroreceptor reflex is a powerful neural feedback that regulates arterial pressure (AP). Mechanosensitive channels transduce pulsatile AP to electrical signals in baroreceptors. Here we show that tentonin 3 (TTN3/TMEM150C), a cation channel activated by mechanical strokes, is essential for detecting AP changes in the aortic arch. TTN3 was expressed in nerve terminals in the aortic arch and nodose ganglion (NG) neurons. Genetic ablation of Ttn3 induced ambient hypertension, tachycardia, AP fluctuations, and impaired baroreflex sensitivity. Chemogenetic silencing or activation of $T \operatorname{tn} 3^{+}$neurons in the NG resulted in an increase in AP and heart rate, or vice versa. More important, overexpression of $T \operatorname{tn} 3$ in the NG of $T \operatorname{tn} 3^{-1-}$ mice reversed the cardiovascular changes observed in Ttn $3^{-/-}$mice. We conclude that TTN3 is a molecular component contributing to the sensing of dynamic AP changes in baroreceptors.
\end{abstract}

\section{Introduction}

Arterial pressure (AP) is maintained at a constant level by cardiovascular reflexes. The baroreflex is a cardiovascular reflex that is essential for blood pressure homeostasis $(1,2)$. It is the most powerful and rapid neural feedback for stabilizing abrupt changes in AP. Thus, the baroreflex is a safeguard to maintain a constant level of AP and consists of 3 components to complete a feedback control system: the baroreceptor afferent nerves, the central controller, and the efferent autonomic nervous system (3-5).

Baroreceptors are end organs scattered throughout the aorta, common carotid artery, and carotid sinus to detect changes in AP (4). The soma of afferent nerves from baroreceptors in the aorta and carotid sinus are located in the nodose ganglion (NG) and petrosal ganglia, respectively $(6,7)$. The nerve endings of baroreceptors located in the adventitia of the aortic arch and carotid sinus sense the stretch of the large vessels in response to high AP (8-10). Afferent signals from baroreceptors in the aorta and carotid sinus are transmitted to the cardiovascular center in the medulla oblongata via the aortic depressor nerve (ADN) and carotid sinus nerve, respectively. An increase in aortic pressure results in an increase in baroreceptor activity, which in turn suppresses sympathetic outflows and augments parasympathetic outflows (3-5). Thus, it decreases the heart rate (HR) and vascular tone, leading to a reflex drop in AP.

Several studies suggested acid-sensing ion channel 2 (ASIC2), transient receptor potential channel 5 (TRPC5), transient recep-

Related Commentary: p. 3412

Conflict of interest: The authors have declared that no conflict of interest exists. Copyright: () 2020, American Society for Clinical Investigation.

Submitted: September 25, 2019; Accepted: March 24, 2020; Published: June 2, 2020.

Reference information: / Clin Invest. 2020;130(7):3671-3683.

https://doi.org/10.1172/JCl133798. tor potential channel subfamily V member 1 (TRPV1), or epithelial sodium channel (ENaC) as molecular identities of mechanosensory channels in the baroreceptor (11-14). Genetic ablation of each gene impairs the baroreceptor's sensitivity, suggesting that multiple mechanosensitive channels might be involved. Recently, Piezo1 and Piezo2, mechanically activated (MA) channels responsible for rapidly adapting (RA) currents observed in dorsal root ganglion neurons, were also found to be essential for baroreceptor function (15). Piezo1 and Piezo2 double-KO mice show reduced baroreceptor activity as well as hypertension and tachycardia, which are hallmarks of baroreceptor denervation (15). This finding adds to the diversity in molecular components of the mechanosensing system in baroreceptors.

An ensemble of channels in the sensory system is often tuned to detect a stimulus over a wide dynamic range. For example, many TRP channels and anoctamin 1 in a subset of somatosensory ganglion neurons detect temperature changes $(16,17)$. Each of these thermo-TRP channels has a distinct temperature range for its activation (18-20). Likewise, multiple mechanically gated channels would detect the AP change in baroreceptors. Recently, a novel MA channel, tentonin 3 (also known as TTN3 or TMEM150C, and referred to hereafter as TTN3), was discovered (21). TTN3 is a cation channel activated by mechanical stimuli in the heterologous system. Its mechanosensitivity is blocked by gadolinium, GsMTx4, and FM1-43, which are largely known as MA channel blockers (21). TTN3 undergoes slow inactivation and is therefore classified as a slowly adapting (SA) MA channel found in dorsal root ganglion neurons (21). TTN3 is expressed in muscle spindle afferents and mediates muscle coordination (21). Surprisingly, we also found a significant expression level of TTN3 in the NG, where the soma of parasympathetic afferent nerves from viscera, including the aorta, lungs, and gastrointestinal tract, are located $(6,22,23)$. Because TTN3 is a MA channel, we hypothesized that TTN3 might be actively involved in detecting AP changes in baroreceptors. 


\section{A}
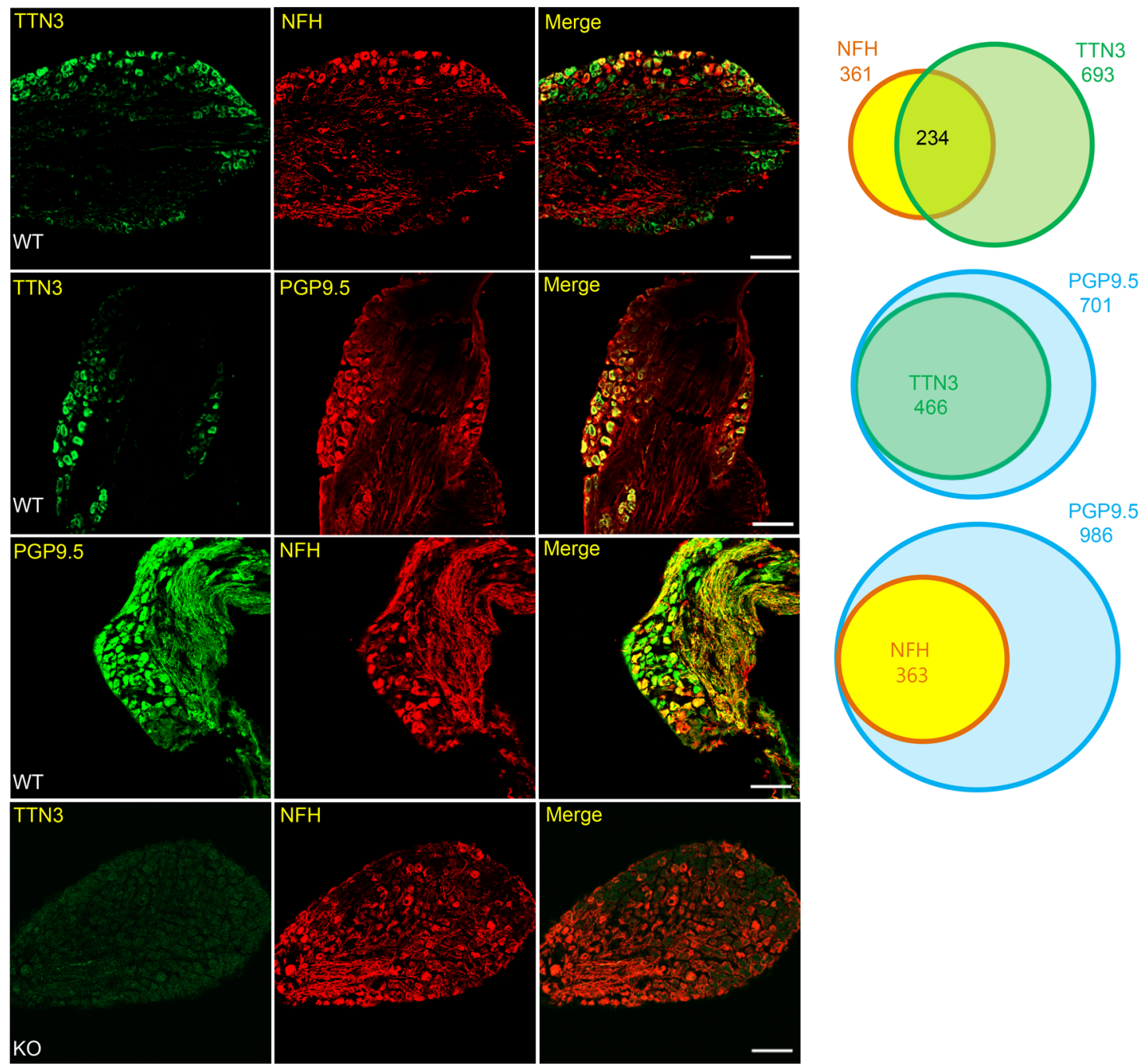

B

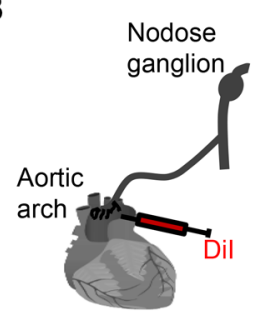

c
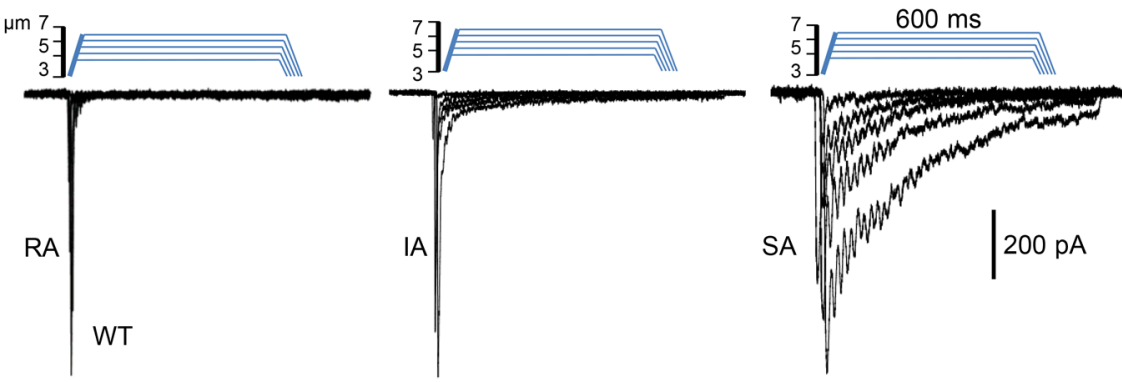

D

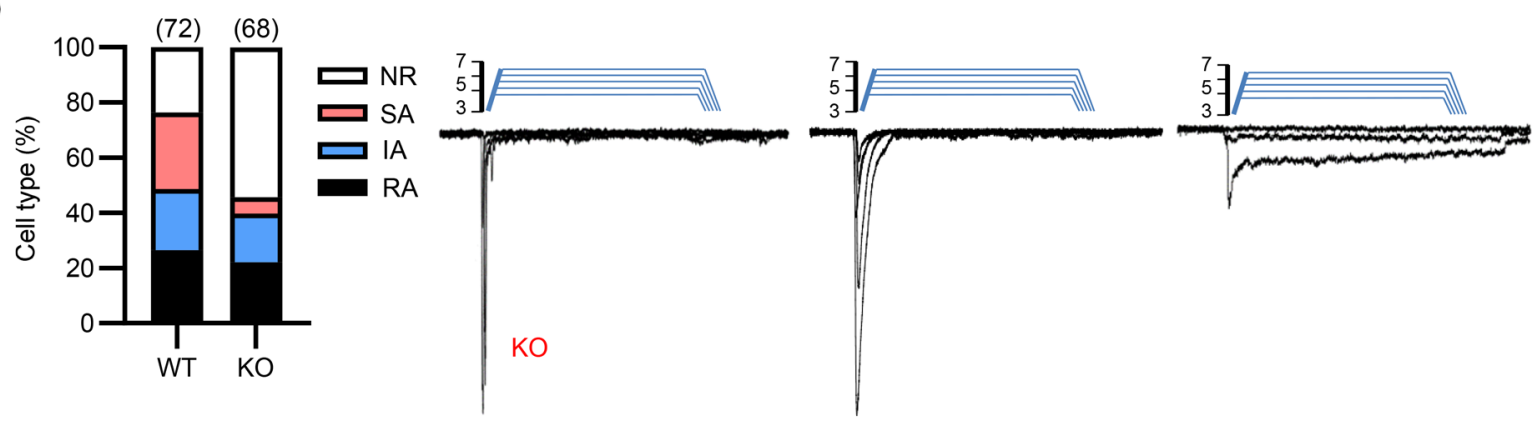


Figure 1. TTN3 is responsible for SA MA currents in aortic archprojecting NG neurons. (A) Immunofluorescence images of TTN3 in NGs isolated from WT and Ttn $^{-1-}$ (KO) mice. NG neurons were stained with anti-TTN3, -NFH, and -PGP9.5 antibodies. Scale bars: $100 \mu \mathrm{m}$. The proportions of colocalization between these markers are shown in the Venn diagrams. (B) Schematic diagram showing injection of the tracer Dil into the aortic arch to selectively label aortic arch-projecting neurons in the NG. (C) Representative traces of 3 types of MA currents in $\mathrm{Dil}^{+}$ NG neurons isolated from WT and KO mice. (D) Summary histogram illustrating 3 types of MA currents characterized in aortic arch-projecting NG neurons based on inactivation kinetics. No response (NR), SA, IA, and RA MA currents are shown. Numbers in parentheses represent the total number of NG neurons tested.

\section{Results}

TTN3 confers MA channel currents in Piezo1-deficient cells. Although TTN3 is activated by mechanical stimuli with distinct inactivation kinetics (21), we sought to identify its gating mechanism. Because heterologous expression of $\operatorname{Ttn} 3$ fails to generate MA currents in Piezo1-KO human embryonic kidney (HEK-P1-KO ) cells, TTN3 is considered a modulator of Piezo1, not an independent MA channel $(24,25)$. However, we observed robust MA currents when Ttn3 was transfected in Piezo1-deficient cells (Supplemental Figure 1A; supplemental material available online with this article; https://doi.org/10.1172/JCI133798DS1). When mechanical strokes were applied to HEK-P1-KO cells transfected with Ttn3, we observed no appreciable MA currents (Supplemental Figure 1A). Because Piezo2 is known to promote actin-based stress fibers in cells (26), weaker cytoskeletal integrity or traction force in HEK-P1-KO cells may lead to loss of the mechanosensitivity of TTN3 in HEK-P1-KO cells. Indeed, when the Ttn3-transfected HEK-P1-KO cells were treated for 2 hours with $250 \mathrm{nM}$ jasplakinolide, an actin-stabilizing agent (27-29), the mechanical strokes robustly evoked MA currents in HEK-P1-KO cells transfected with Ttn3 (Supplemental Figure 1, A and B). In addition, when HEK cells expressing Ttn 3 were treated with $5 \mu \mathrm{M}$ cytochalasin D, an actin-cytoskeleton disruptor $(30,31)$, the mechanosensitivity of TTN3 was blocked completely (Supplemental Figure 1, C and D). These results clearly indicate that the mechanosensitivity of TTN3 is largely dependent on cytoskeletal integrity, which might be low in Piezo1-deficient cells.

TTN3 confers SA MA currents in NG neurons. We examined the presence of TTN3 in the NG, where the somas of aortic baroreceptors are located. Transcripts of Ttn3 were present in NG cells, as shown by PCR analysis (Supplemental Figure 2A). We stained sections of mouse NG with TTN3 antibody; neurofilament heavy chain (NFH), a marker for myelinated axons; and protein gene product 9.5 (PGP9.5), a sensory neuron-specific marker $(32,33)$. We found that TTN3 was expressed in the majority of NG neurons, because approximately $66.5 \%$ of PGP9.5 $5^{+}$neurons were colocalized with TTN3 (Figure 1A). In addition, $33.7 \%$ of TTN3 ${ }^{+}$neurons were also positive for NFH. Approximately $36.8 \%$ of PGP9.5 neurons were also positive for $\mathrm{NFH}$, suggesting that the majority of NG neurons are probably unmyelinated neurons. We also confirmed the expression of Piezo2 in NG neurons. Interestingly, the majority $\left(87.8 \%\right.$ ) of TTN3 ${ }^{+}$neurons contain Piezo2 (Supplemental Figure 2, B and C). However, we did not observe TTN $3^{+}$neurons in the NGs of $\operatorname{Ttn~}^{-/-}$mice (Figure 1A). Moreover, the gross histologi- cal structures of the aorta and left ventricle of $\mathrm{Ttn}^{-/-}$mice showed no impairment when compared with those of WT mice (Supplemental Figure 3, A and B).

Because neurons in the NG contain somas of parasympathetic afferent nerves from all viscera including the aorta, lungs, and gastrointestinal tract $(6,22,23)$, NG neurons that innervate in the aortic arch were retrogradely labeled with a fluorescent dye, DiI. We injected the dye into the adventitia of the aortic arches (Figure 1B). After 7 days of recovery, the NG neurons were primary cultured and stained with the TTN3 antibody. We found DiI ${ }^{+}$ neurons in 9.9\% of NG neurons (Supplemental Figure 4, A and B). In addition, approximately $74.8 \%$ of these baroreceptor neurons had TTN3 immunoreactivity (Supplemental Figure 4B). These results indicate that TTN3 is expressed in NG neurons that innervate in the aortic arch.

TTN3 is an MA channel with slow inactivation kinetics (21). Therefore, if TTN3 is present in aortic baroreceptors, $\mathrm{DiI}^{+} \mathrm{NG}$ neurons should retain MA currents with slow inactivation. We therefore applied mechanical pulses (2.5- to 7.2- $\mu \mathrm{m}$ indentation for 600 $\mathrm{ms})$ to $\mathrm{DiI}^{+} \mathrm{NG}$ neurons. These mechanical step stimuli evoked 3 different types of MA currents. Depending on the inactivation kinetics, the MA currents in $\mathrm{Dil}^{+} \mathrm{NG}$ neurons could also be categorized into 3 groups, as observed in dorsal root ganglion neurons: RA currents $\left(\tau_{\mathrm{i}}=4.3 \pm 0.5 \mathrm{~ms}, n=19\right)$, intermediately adapting (IA) currents $\left(\tau_{\mathrm{i}}=22.1 \pm 1.7 \mathrm{~ms}, n=16\right)$, and SA currents $\left(\tau_{\mathrm{i}}=127.9 \pm 21.8\right.$ $\mathrm{ms}, n=20$ ) (Figure 1, C and D). Among the 72 aorta-innervated NG neurons, 26.4\% were RA-type, 22.2\% were IA-type, and 27.8\% were SA-type neurons (Figure 1D). The other neurons $(23.6 \%$ of the tested neurons) showed no response to the mechanical stimuli. We then repeated testing of the mechanosensitivity of Dil ${ }^{+}$ NG neurons isolated from $\mathrm{Ttn}^{-/-}$mice. The proportion of neurons with SA currents was markedly reduced to $5.9 \%$ (Figure 1D). The proportion of $\mathrm{Dil}^{+} \mathrm{NG}$ neurons with no response to the mechanical stimuli increased in $\mathrm{Ttn}^{-/}$mice, which might be due to a reduction in the SA NG neurons. Notably, the amplitude of SA currents observed in NG neurons was also remarkably reduced (Figure 1C). These results suggest that TTN3 functions as a SA MA channel in aortic baroreceptors.

TTN3 is expressed on the ADN in the aortic arch. We next determined whether TTN3 is localized at the terminals of the ADN in the aortic arch. To visualize $\mathrm{TTN}^{+}$nerve fibers in the ADN, we expressed enhanced yellow fluorescent protein (EYFP) on the Ttn3 promoter in NG neurons. To do this, we generated Cre-transgenic mice at the Ttn 3 promoter region (Ttn $3^{\mathrm{Cre}}$ mice). An adenoassociated virus (AAV) carrying EF1 $\alpha$-DIO-EYFP (AAVDJ-EF1 $\alpha$ DIO-EYFP) was injected into the left NG of Ttn $3^{\mathrm{Cre}}$ mice (Figure 2A). To visualize the nerve terminals in the aorta, a piece of the aortic arch was cut, fixed with paraformaldehyde (PFA), cleared with electroporation, and stained with anti-EYFP and anti-NFH antibodies $(34,35)$. As shown in Figure 2, B and C, the EYFP nerve fibers were spread over the adventitia of the aorta, colocalizing with NFH. The $\mathrm{EYFP}^{+}$fibers were coiled and tortuous in a complex manner, such that they formed a nerve plexus and arborized along the adventitia of the aorta (Supplemental Video 1). We also injected AAVDJ-EF1 $\alpha$-DIO-mCherry into the NGs of Ttn $3^{\text {Cre }}$ mice to express mCherry in $\mathrm{TTN}^{+}$nerves in the aorta. Thin sections of the aorta revealed 2 types of nerve terminals labeled with 
A

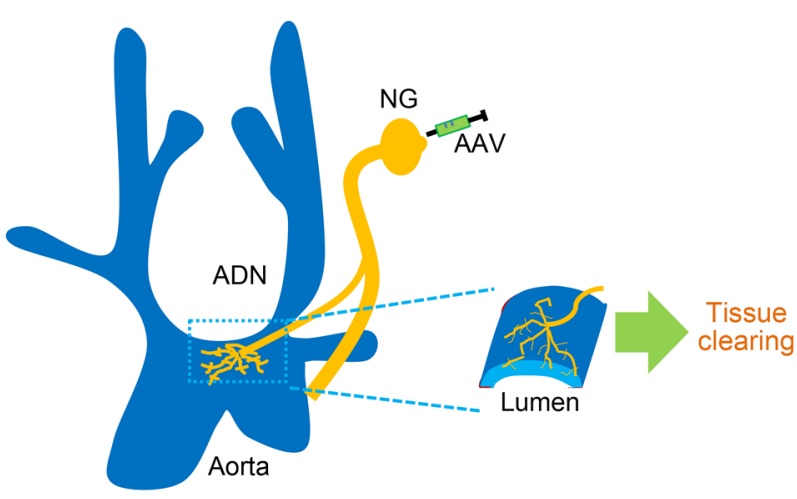

B

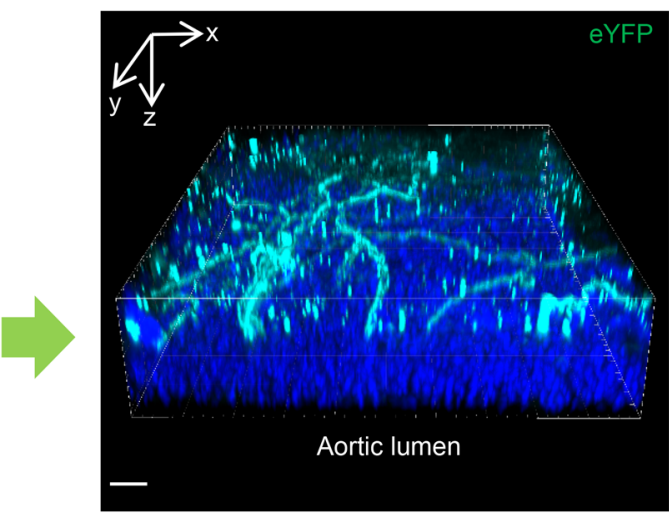

C

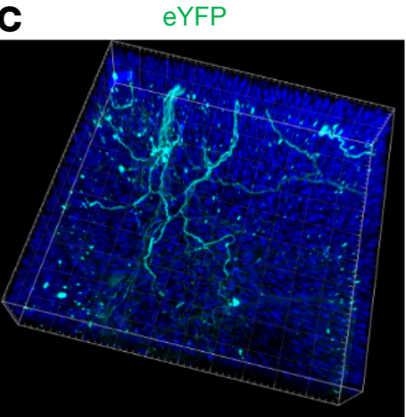

D

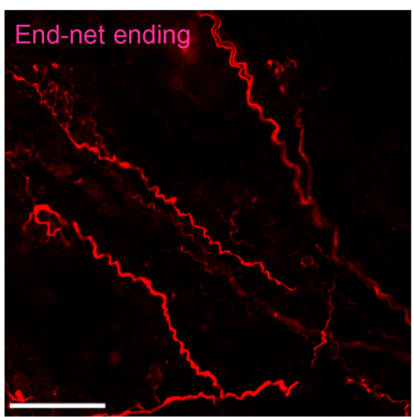

$\mathrm{NFH}$

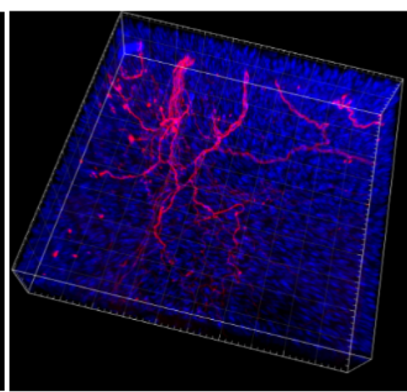

Merge
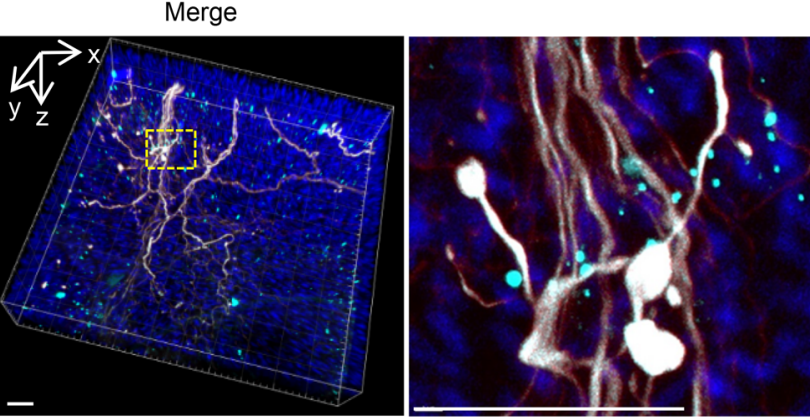

E

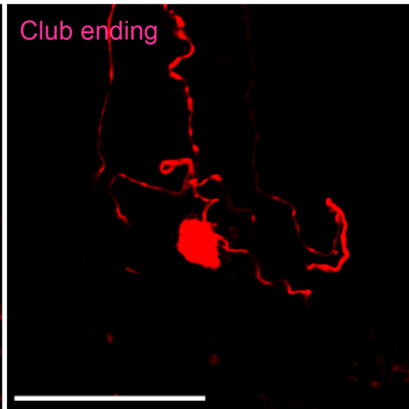

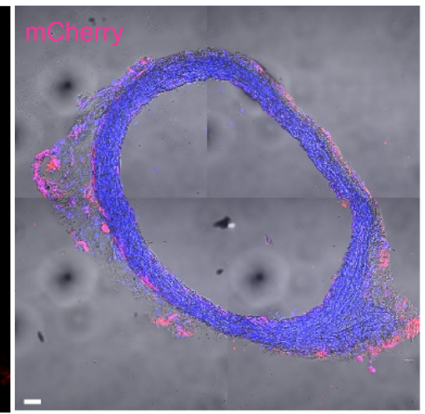

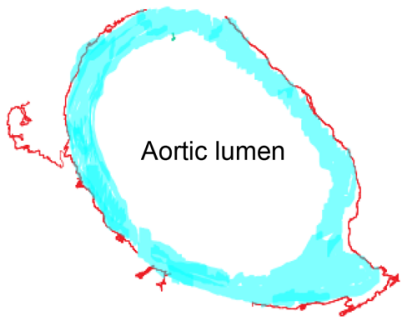

Figure 2. TTN3 is present in the nerve terminals of the ADN. (A) Schematic illustration of tissue clearing and imaging of the aortic arch where ADN attaches. AAVDJ-EF1 $\alpha$-DIO-EYFP (B and C) or AAVDJ-EF1 $\alpha$-DIO-mCherry (D and E) was injected into the NGs of Ttn3 ${ }^{\text {Cre }}$ mice to label TTN3 ${ }^{+}$nerve terminals in the aortic arch. (B) 3D-reconstructed images of ADN terminals in the aortic arch adventitia stained with EYFP antibody and DAPI. (C) 3D-reconstructed images of ADN terminals stained with EYFP and NFH. The far-right panel shows the magnified image of the yellow dashed box in the merged panel. (D) mCherry ${ }^{+}$nerve terminals: end-net endings and club ending. (E) Ensemble cross-sectional image depicting the whole aorta. Note that the mCherry ${ }^{+}$nerve terminals surround the entire aorta (left). Schematic illustration on the right depicts the image shown in the left panel. Scale bars: $50 \mu \mathrm{m}$.

mCherry: "end-net" endings that showed thin and long "free" nerve endings and "discoid or club" endings, where terminals stopped abruptly with an enlargement (Figure 2D). These specialized nerve terminals have been described as baroreceptors in the aortic arch in rats and mice (36-39). Ensemble images of the cross section of the whole aorta showed that $\mathrm{TTN}^{+}$fibers encircled the entire aorta (Figure 2E). These results show that TTN3 is expressed at baroreceptor nerve terminals where the mechanotransduction occurs and that these specialized morphologies of the nerve terminals are probably suitable for the detection of pressure changes in the aorta.

TTN3 is required for pressure-evoked action potentials in the ADN. Because TTN3 is a MA channel expressed in nerve terminals of the ADN, TTN3 would be functionally active in sensing pressure changes in the aortic arch. We therefore isolated the aortic arch along with the ADN attached to the aorta and recorded the neural activity of the ADN ex vivo. In this preparation, pressurized saline was injected into the thoracic end of the aorta, while the cardiac end of the aorta and arterial branches were tied (Figure 3A). When the aortic arch was pressurized at 50,100, and $150 \mathrm{mmHg}$, the nerve fibers of the ADN from mice of both genotypes discharged action potentials in response to the intra-aortic pressures (Figure 3, B-D). The nerve activity of the ADN from WT mice increased as the pressure increased from 0 to 50 to 100 to $150 \mathrm{mmHg}$ (Figure 3, B-D). In contrast, the ADN activity in Ttn $3^{-/}$mice was markedly reduced at 100 and 150 mmHg compared with that observed in WT mice (Figure 3, B-D). 
A

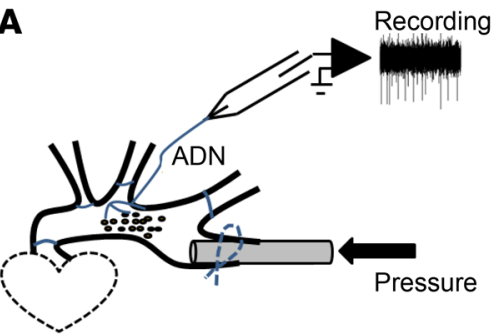

Figure 3. TTN3 is required for pressure-evoked action potentials in the ADN. (A) Schematic illustration of ex vivo recording from an isolated ADN attached to the aortic arch. (B) Representative traces of multiunit activities in response to intraaortic pressures in WT and $\mathrm{Ttn}^{-1-}$ mice. Aortic pressure is shown in the middle panel. The bottom panel shows the number of spikes per second. ADNA, ADN activity. (C) Representative traces of ADN activity at intra-aortic pressures of $0,50,100$, and 150 $\mathrm{mmHg}$ in an expanded time scale. (D) Summary of ADN activity in WT $(n=12)$ and $\operatorname{Ttn}^{-1-}(\mathrm{KO})(n=7)$ mice in response to the intra-aortic pressures. Data represent the mean \pm SEM. ${ }^{* *} P<0.01$ and ${ }^{* *} P<0.001$, by Student's $t$ test.

\section{B}

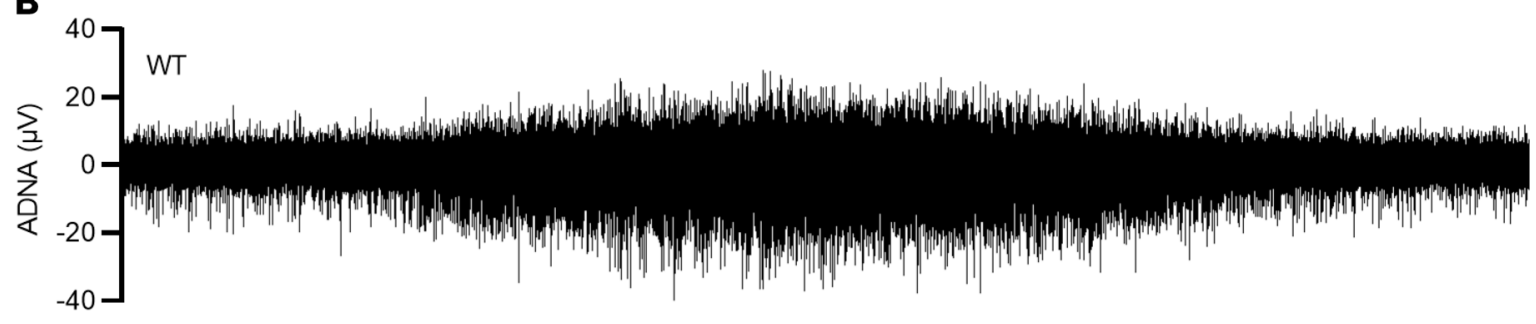

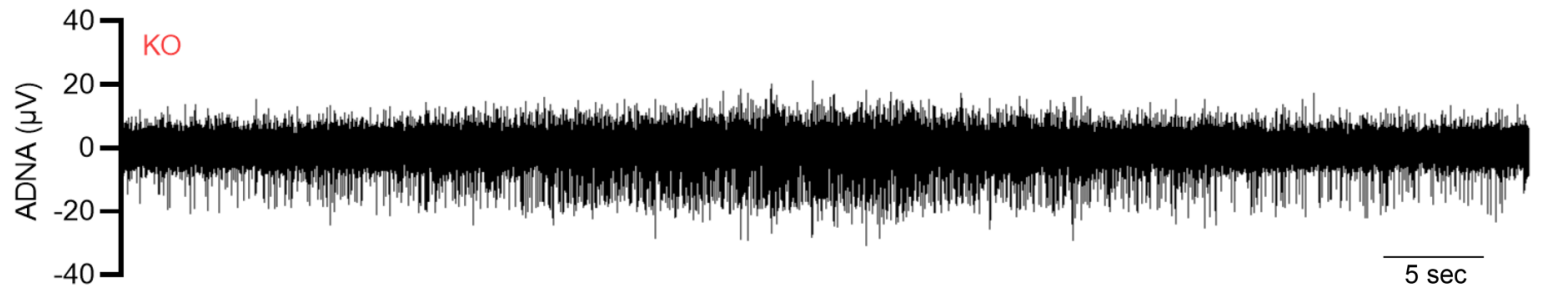

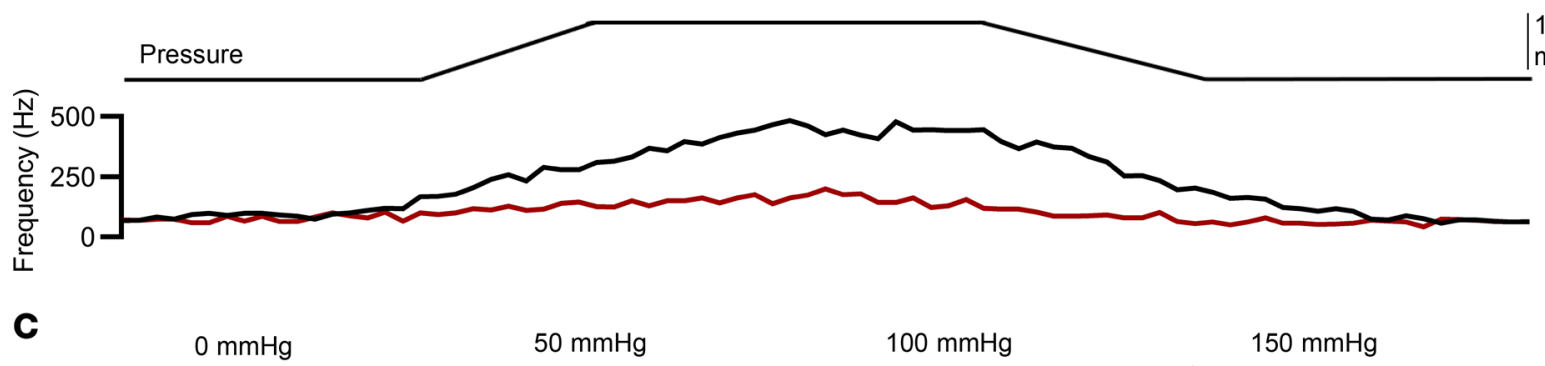

WT
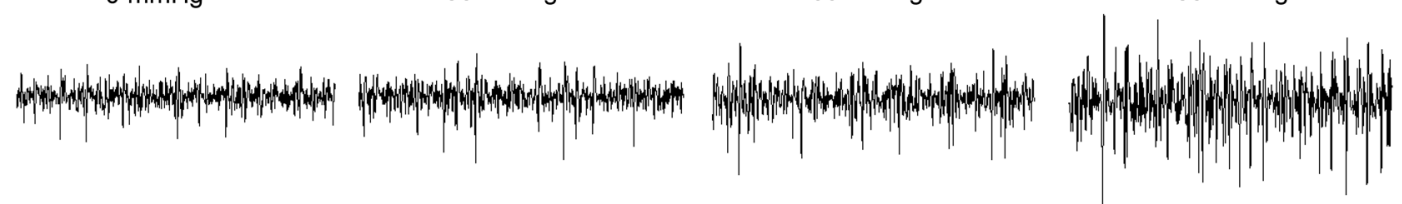

$\mathrm{KO}$
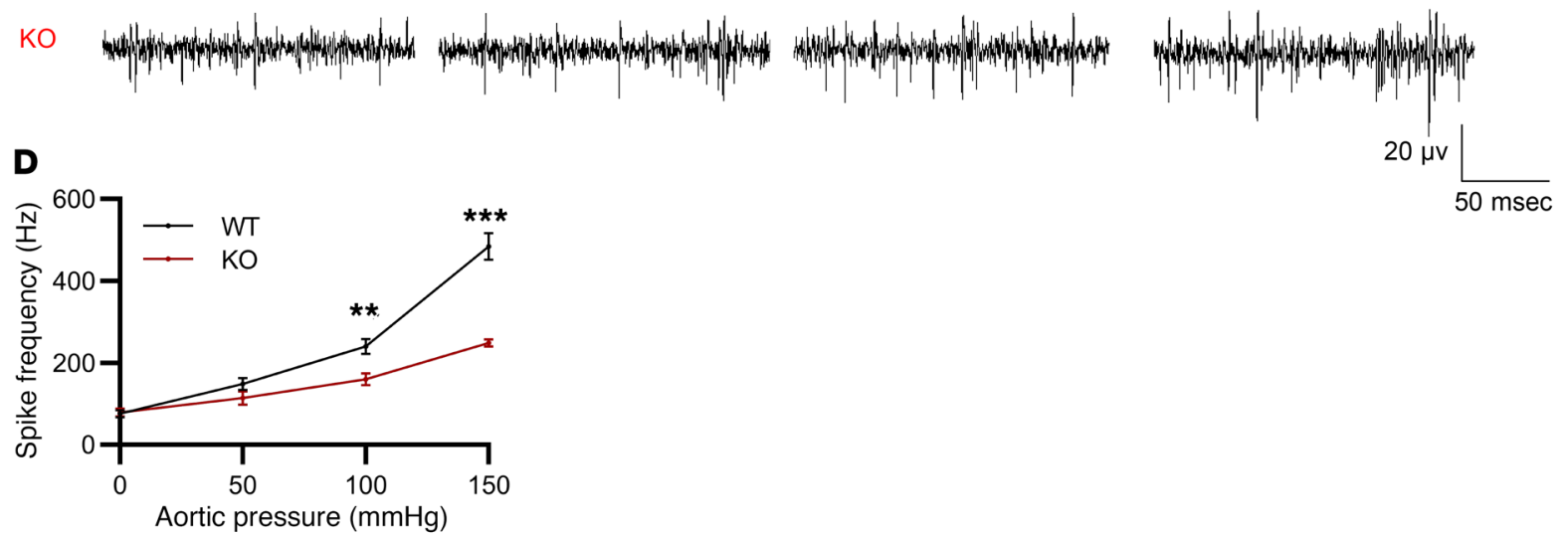

Genetic ablation of Ttn3 induces hypertension, tachycardia, and AP instability. If TTN3 acts as a molecular sensor to detect a pressure change in baroreceptors, the genetic ablation of Ttn3 would affect AP and HR. We therefore recorded ambient APs and HRs of freely moving mice of both genotypes for 24 hours continuously using a telemetric device. The 24-hour ambient APs of WT mice were stable throughout the duration of the recording (Figure 4A). Notably, APs changed diurnally, with higher APs recorded at night 
A

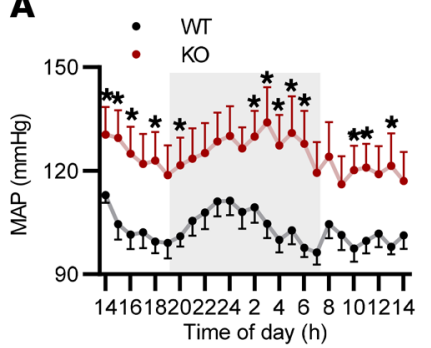

E
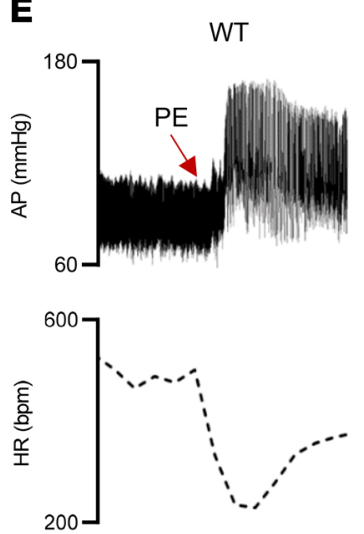

B

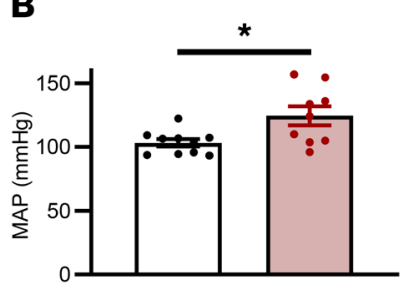

$\mathbf{F}$
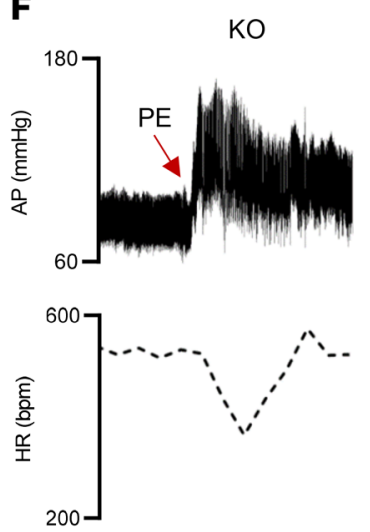

C

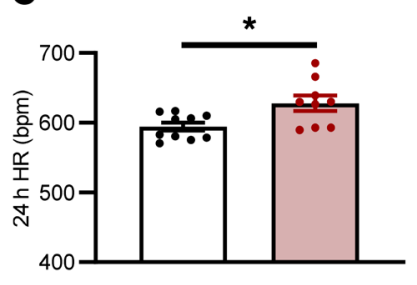

G

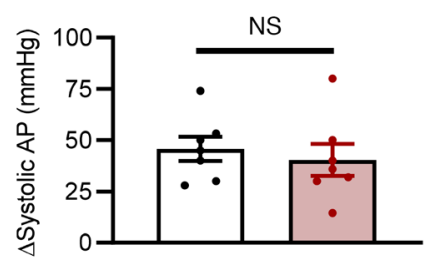

H

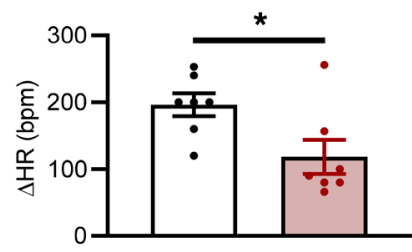

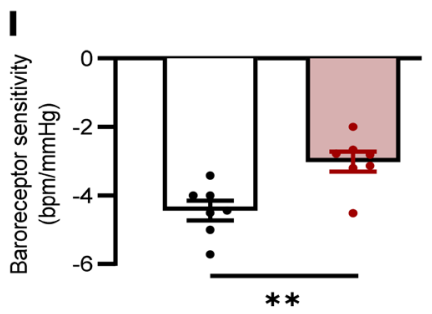

Figure 4. Ttn3 ablation results in hypertension, tachycardia, AP instability, and reduced baroreflex sensitivity. (A) Continuous recordings of MAP in WT $(n=10)$ and $\operatorname{Ttn}^{-/-}(n=9)$ mice over a 24-hour period. A telemetric device was embedded before recording ambient AP in freely moving mice. Recordings were made every 5 minutes. The shaded area represents nighttime. ${ }^{*} P<0.05$, by Student's $t$ test. (B and C) Summarized data of average MAP (B) and HR (C) over a 24-hour period in WT $(n=10)$ and $\operatorname{Ttn}^{-/-}(n=9)$ mice. Data represent the mean \pm SEM. ${ }^{*} P<0.05$, by Student's $t$ test. (D) Frequency distribution histogram of MAP over a 24-hour period in WT $(n=10)$ and Ttn3 $3^{-/-}(n=9)$ mice. (E and F) Representative traces of the changes in AP and HR in response to an intravenous injection of PE in conscious WT $(n=7)(\mathbf{E})$ and $\operatorname{Ttn}^{-/-}(n=7)(\mathbf{F})$ mice. (G and H) Average changes in systolic arterial pressure (SAP) $(\Delta S A P)$ (C) and heart rate $(\triangle H R)(H)$ in response to an intravenous injection of PE in WT and Ttn $3^{-/-}$mice ( $n=7$ mice per group). Data represent the mean \pm SEM. ${ }^{*} P<0.05$, by Student's $t$ test. (I) Baroreflex sensitivity $(\Delta \mathrm{HR} / \Delta \mathrm{SAP})$ after the intravenous PE injection in WT and Ttn3 ${ }^{-/-}$mice $(n=7$ mice per group). Data represent the mean \pm SEM. ${ }^{*} P<0.01$, by Student's $t$ test.

than during the daytime because of the higher locomotor activity at night (Supplemental Figure 5, A and B). Diurnal changes in APs in $\operatorname{Ttn}^{-1-}$ mice were similar to those in WT mice. However, as expected for baroreceptor denervation (40), the overall APs in the 24-hour recording were significantly higher (Figure 4, A and B). The mean AP (MAP) of $\operatorname{Ttn}^{-/-}$mice was significantly higher than that of WT mice $(124.4 \pm 7.4$ vs. $103.2 \pm 2.9 \mathrm{mmHg}$ ) (Figure 4B). The mean HR of $\operatorname{Ttn}^{-1-}$ mice was also higher than that of WT mice $(627.9 \pm 11.1$ vs. $594.1 \pm 5.7 \mathrm{bpm})$ (Figure 4C). More important, $\operatorname{Ttn}^{-/-}$mice elicited a larger distribution of MAPs than did WT mice (Figure 4D). These parameters are a strong indication of baroreceptor denervation (41). In contrast, we observed no significant differences in 24-hour locomotor activity between WT and Ttn3 $^{-/-}$mice (Supplemental Figure 5, A and B).

Genetic ablation of Ttn3 impairs baroreflex sensitivity but not the central controller of the baroreflex. We next tested whether genetic deletion of Ttn 3 could lead to changes in baroreflex sensitivity in vivo. The gain of baroreflex sensitivity was calculated by the ratio of the change in HR to the changes in systolic AP $(\Delta \mathrm{HR} / \Delta \mathrm{SAP})(12,42)$. A bolus injection of phenylephrine (PE), an $\alpha$-adrenergic receptor agonist, into the femoral vein of WT mice increased AP rapidly with a concomitant reflex decrease in the HR (Figure 4, E and F). The average increase in systolic arterial pressure of $\mathrm{Ttn}^{-/-}$mice after the PE injection was comparable to that of the WT mice $(40.4 \pm 7.8$ vs. $45.4 \pm 5.1 \mathrm{mmHg})$ (Figure 4G). However, the reflex decrease in the HR in Ttn3 $3^{--}$ mice was significantly lower than that of the WT mice (118.4 \pm 25.5 vs. $196.2 \pm 17.1 \mathrm{bpm}$ ) (Figure $4 \mathrm{H}$ ). Therefore, the baroreflex sensitivity of $\mathrm{Ttn}^{-/-}$mice was significantly less than that of WT mice (Figure 4I). These results indicate an active role of TTN3 in mediating the baroreflex in vivo.

We hypothesized that genetic ablation of Ttn3 might affect the central controller of the baroreflex. To test this, we electrically stimulated the ADN in anesthetized mice. As shown in Figure $5 \mathrm{~A}$, electrical stimulation of the ADN induced a reflex decrease in MAP and HR in WT mice. The reflex decrease in MAP and HR in WT mice was proportional to the frequency of the ADN stimulation. Similarly, electrical stimulation of the ADN in $\mathrm{Ttn}^{-/-}$mice induced a reduction in MAP and HR almost identical to that observed in WT mice (Figure 5, A-C). These results suggest that the central controller function of $\operatorname{Ttn~}^{-/-}$mice is not affected by genetic ablation of Ttn3.

Chemogenetic inhibition or stimulation of $\mathrm{TTN}^{+}$neurons in the NG induces hypertension or hypotension, respectively. We next 
A
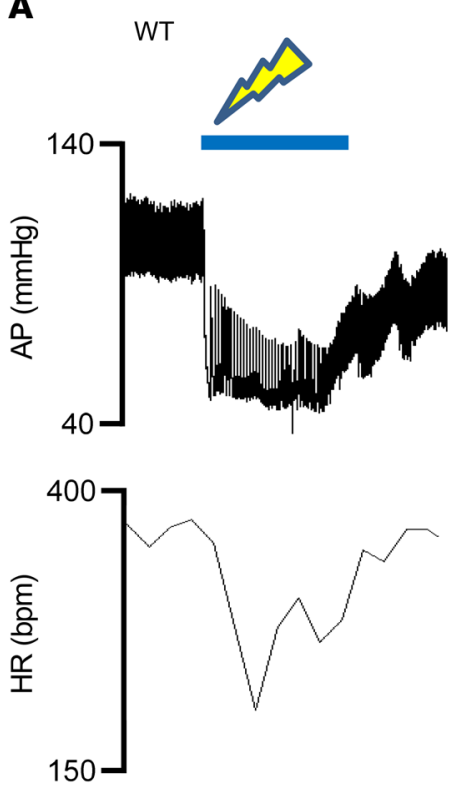

KO
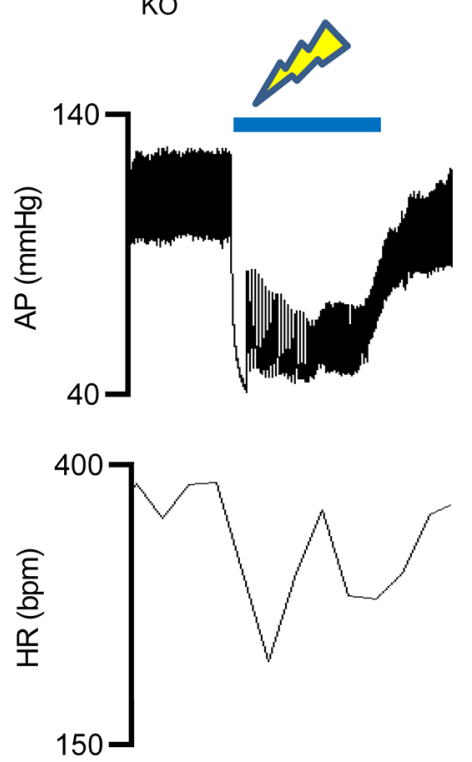

B

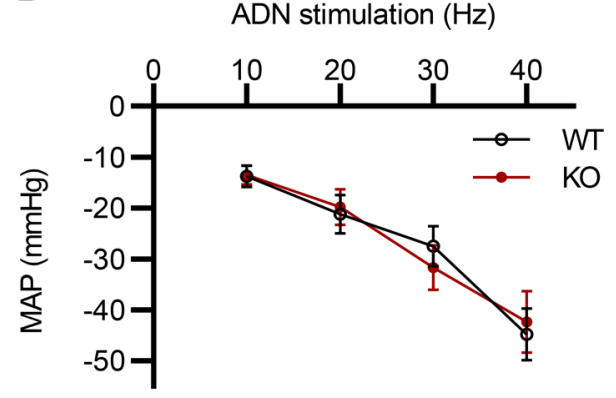

$c$

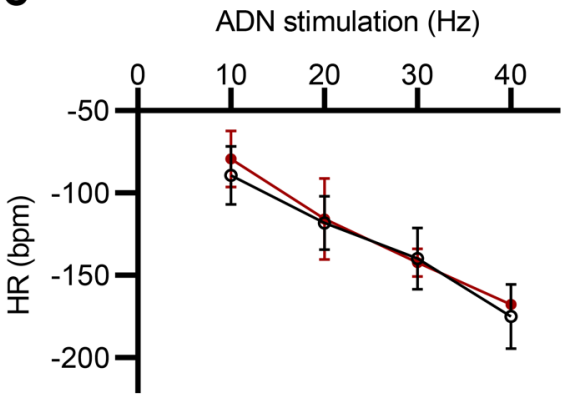

Figure 5. Effect of electrical stimulation of the ADN on MAP and HR in anesthetized WT and Ttn $3^{-/-}$mice. (A) Representative traces of the rapid decrease in AP (top) and HR (bottom) under $40-\mathrm{Hz}$ electrical stimulation for 20 seconds (blue bar) in a WT and a Ttn3 ${ }^{-/-}$mouse. (B and C) Summaries of MAP (B) and $\mathrm{HR}(\mathbf{C})$ in response to electrical stimulation of the ADN. $n=6$ WT mice; $n=6 \mathrm{Ttn}^{-1-}$ mice. Data represent the mean \pm SEM.

investigated whether selective inhibition or activation of $\mathrm{TTN}^{+}$ neurons in NG can affect the baroreflex in vivo. We used the designer receptor exclusively activated by designer drug (DREADD) system to test our hypothesis $(43,44)$. We injected AAVhsyn-DIO-mCherry, AAV-hsyn-DIO- $h M 4 D i$-mCherry or AAVhsyn-DIO- $h M 3 D q$-mCherry into the NGs of $T \operatorname{tn} 3^{\text {Cre }}$ mice. These viruses ensure mCherry, hM4Di, or hM3Dq expression only in Creexpressing NG neurons. hM4Di and hM3Dq are artificially designed GPCRs that stimulate Gi or Gq proteins, respectively, to induce inhibition or excitation of neurons upon clozapine- $N$-oxide (CNO) application $(43,44)$. Postmortem analysis revealed that the viral infection of the 3 types of constructs expressed mCherry in NG neurons (Supplemental Figure 6). Four weeks after the viral infection, AP and HR were recorded with a telemetric pressure probe in freely moving mice. Saline was injected twice daily for 3 days for habituation before the CNO injection to reduce cardiovascular changes caused by a psychological disturbance in response to intraperitoneal injection of $\mathrm{CNO}$. Intraperitoneal injection of CNO into the mice infected with AAV-hsyn-DIO-hM4DimCherry induced a significant increase in the MAP and HR (11.9 \pm 1.7 vs. $0.6 \pm 2.7 \mathrm{mmHg}, 48.7 \pm 15.1$ vs. $6.7 \pm 4.8 \mathrm{bpm}$ ), measured for 40 minutes after the CNO injection (Figure 6), compared with the MAP and HR of mice infected with AAV-hsyn-DIO-mCherry. Conversely, CNO injection into the mice infected with AAV-hsynDIO- $h M 3 D q$-mCherry induced a significant decrease in the MAP and $\operatorname{HR}(-14.7 \pm 1.07$ vs. $0.6 \pm 2.7 \mathrm{mmHg},-40.1 \pm 7.8$ vs. $6.7 \pm 4.8$ bpm) (Figure 6). These results further suggest that $\mathrm{TTN}^{+} \mathrm{NG}$ neurons contribute to baroreflex function.

Overexpression of TTN3 in the NG of $\mathrm{Ttn}^{-1-}$ mice rescues the impaired baroreceptor. To confirm the role of TTN3 in barorecep- tor function, we overexpressed $\operatorname{Ttn} 3$ in the NG of $\mathrm{Ttn}^{-/-}$mice to see whether the impaired baroreceptor function was rescued. We injected AAV containing either mCherry (AAV-hsyn-mCherry, control KO group) or the Ttn3-mCherry gene (AAV-hsyn-Ttn3mCherry, rescue group) into the $\mathrm{NG}$ of $\operatorname{Ttn}^{-1-}$ mice. We observed robust expression of mCherry, with high efficiency in both AAVhsyn-mCherry- and AAV-hsyn-Ttn3-mCherry-infected NGs (72.4\% $\pm 3.6 \%$ vs. $68.7 \% \pm 5.1 \%$, respectively) of $\mathrm{Ttn}^{-/-}$mice (Figure 7, A and B). With the telemetry device, the APs and HRs of mice in the control $\mathrm{KO}$ and rescue groups were recorded for 24 hours. As shown in Figure 7, C-E, the control KO group showed a significant increase in 24-hour MAP, HR, and range of APs compared with WT mice. In contrast, the rescue group had a significant reduction in MAP, HR, and range of APs to the levels detected in WT mice (Figure 7, C-E). In addition, the rescue group regained baroreflex sensitivity to the level seen in the WT group (Figure $7 F$ ). The cardiovascular parameters of WT mice in Figure 4 are presented for comparison. These results confirm that TTN3 plays an essential role in baroreceptor function.

\section{Discussion}

The baroreflex is one of the control mechanisms that regulate AP in normal life $(1,2)$. It is a powerful and fast neural control mechanism of AP (4) and depends on baroreceptor activity in the aortic arch and carotid sinus, which sense AP changes and send neural signals to the central cardiovascular center. Sensing pressure changes in the arteries requires the activity of molecular sensors in baroreceptors in the aortic arch or carotid sinus. The present study shows evidence that TTN3, a newly discovered MA channel, functions as a mechanotransduction channel in baroreceptors. We base this on 


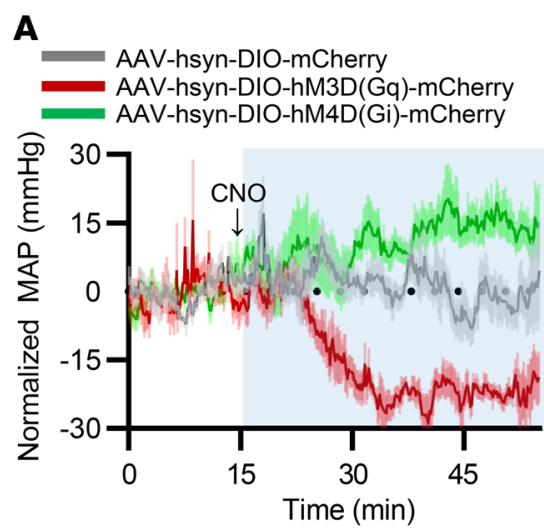

B
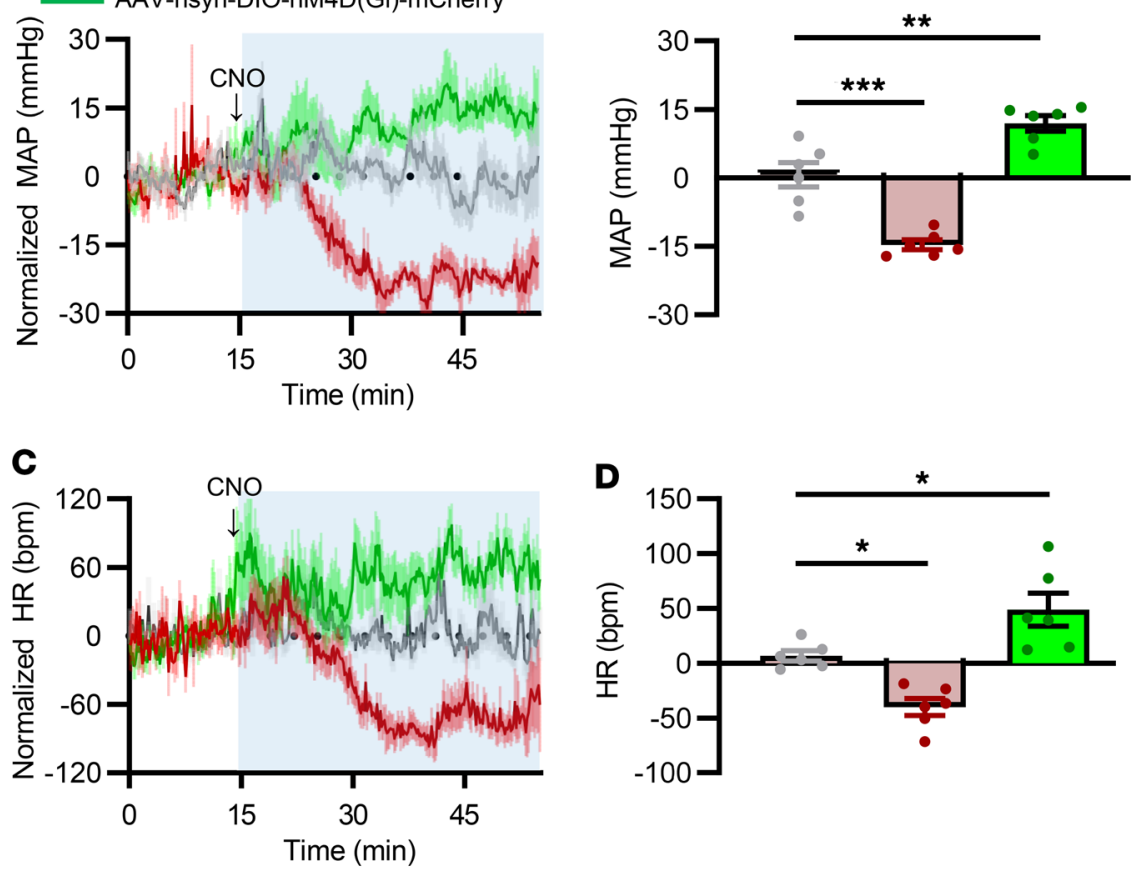

Figure 6. Chemogenetic inhibition or activation of TTN3 ${ }^{+}$NG neurons causes an increase in MAP and HR, or vice versa. ( $A$ and $\mathbf{C}) \operatorname{MAP}(\mathbf{A})$ and $H R(C)$ responses to intraperitoneal injection of $\mathrm{CNO}$ into Ttn ${ }^{\text {Cre }}{ }^{2}$ mice infected with either AAV-hsyn-DIOmCherry (gray), AAV-hsyn-DIO-hM3Dq-mCherry (red), or AAV-hsyn-DIO-hM4Di-mCherry (green). Four weeks after the viral infections, the MAP and $\mathrm{HR}$ were recorded with a telemetric device in freely moving mice. Data were normalized to the baseline MAP or HR obtained from 0 to 15 minutes before the CNO injection (arrows). (B and $\mathbf{D})$ Averages of MAP and $\mathrm{HR}$ changes for $\mathbf{4 0}$ minutes after CNO injection ( $n=6$ mice per group). Data represent the mean \pm SEM. ${ }^{*} P<0.05,{ }^{* *} P<0.01$, and ${ }^{* *} P<0.001$, by 1 -way ANOVA with Dunnett's post hoc test. the following observations: (a) expression of TTN3 in NG neurons as well as in the terminals of the ADN innervating the aortic arch; (b) absence of SA MA currents in aorta-projecting NG neurons of $\mathrm{Ttn3}^{-/}$mice; (c) reduced basal and pressure-induced ADN activity in $\mathrm{Ttn}^{-/-}$mice; (d) increased MAP, HR, and MAP fluctuation in $\mathrm{Ttn}^{-/-}$mice; (e) rescue of impaired baroreceptor function after overexpression of $\operatorname{Ttn} 3$ in the NG of $\operatorname{Ttn~}^{-/-}$mice; and (f) decreased or increased MAP and HR following chemogenetic stimulation or inhibition, respectively, of TTN3-expressing neurons in the NG. Importantly, we also confirmed the mechanosensitivity of TTN3 in Piezo1-deficient cells, arguing against the Piezo1-dependent mechanosensitivity of TTN3 (25). The identification of molecular sensors in baroreceptors can provide a useful clue to treat cardiovascular diseases. However, this study also has some technical limits. The whole-nerve recordings of ADNs failed to detect all spikes in the nerve because of high discharge rates. Also, the chemogenetic study may include indirect effects on the cardiovascular functions that originate in vagal afferents from nonbaroreceptor areas.

Denervation of the ADN or carotid sinus nerve is known to result in acute hypertension, tachycardia, and larger fluctuations of APs in dogs, rabbits, rodents, and humans (41,45-47). Bilateral denervation of the carotid sinus leads to chronic hypertension in humans and other animals except for dogs, whose denervation of aortic baroreceptors only leads to short-term hypertension (41, 45-47). Among the cardiovascular changes, variability in AP after baroreceptor denervation is more consistent. Thus, stabilization of AP appears to be a primary function of baroreceptors. Consistent with this, compared with WT mice, we also observed markedly high MAP, HR, and AP variability was also observed in $\mathrm{Ttn} 3^{-/-}$mice, whose abnormalities were rescued when $\mathrm{Ttn} 3$ was overexpressed in the NG of $\operatorname{Ttnh}^{-/-}$mice. The hypertension and tachycardia observed in $\mathrm{Ttn}^{-/-}$mice could be induced by dysfunction in the central cardiovascular center, but tachycardia was less likely to happen, because electrical stimulation of the ADN led to a reduction in MAP and HR that was comparable to that seen in WT mice (Figure 5). Thus, the phenotype of Ttn 3 ablation resulted from the dysfunction in the baroreceptor afferents, not in the central cardiovascular center. This assumption is also supported in part by the rescue experiment or chemogenetic modulation of $\mathrm{TTN}^{+}$neurons in the NG, because overexpression of Ttn3, hM3Dq, or hM4Di in the NG, but not in the brain, was sufficient to rescue or modulate the baroreflex.

Surprisingly, the MA currents in aorta-projecting NG neurons are similar to those found in dorsal root ganglion neurons $(21,48)$. This study is the first to our knowledge to characterize MA currents in baroreceptor neurons. In response to step mechanical stimuli, we detected 3 different MA currents: RA, IA, and SA currents (Figure 1, C and D). In dorsal root ganglion neurons, SA currents are known to be mediated by TTN3, whereas Piezo2 is responsible for RA currents $(21,48)$. Consistent with somatosensory neurons, SA MA currents were markedly diminished in $\mathrm{Ttn}^{-/} \mathrm{NG}$ neurons. In addition, a large proportion of aorta-projecting NG neurons also elicited RA MA currents (Figure 1, C and D). The presence of RA MA currents in NG neurons suggests a role of Piezo1 or Piezo2 in baroreceptor function. Indeed, Zeng and colleagues reported that Piezo1 and Piezo2 mediate baroreceptor function (15). Piezo2 is expressed in NG neurons. Piezo1 and Piezo2 double-KO mice show hypertension, tachycardia, and loss of baroreceptor sensitivity (15). Piezo1 and Piezo2 appear to be essential for sensing AP changes in baroreceptors, which largely overlaps with the functional role of TTN3 in baroreceptors. Perhaps these findings indicate that a 
A
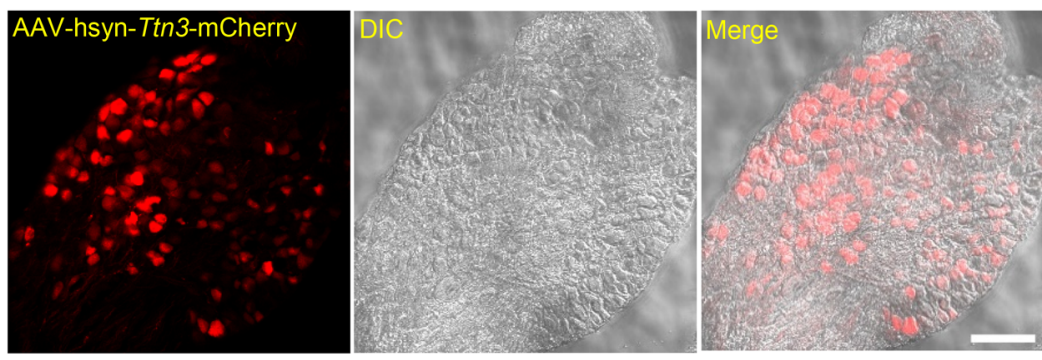

C

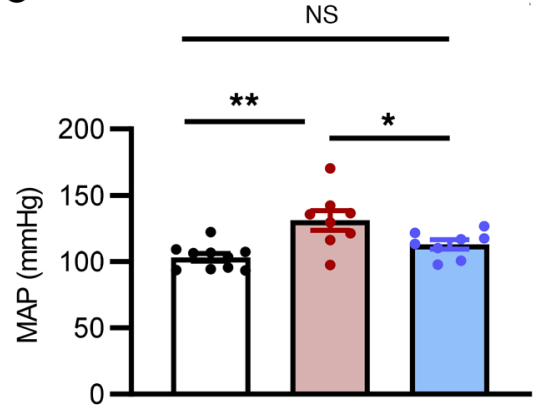

D

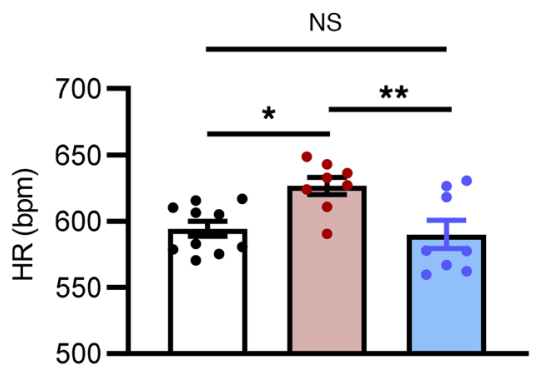

B

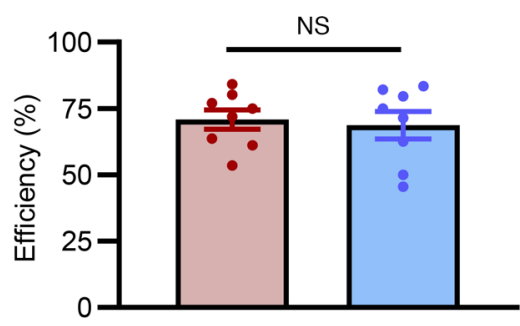

E

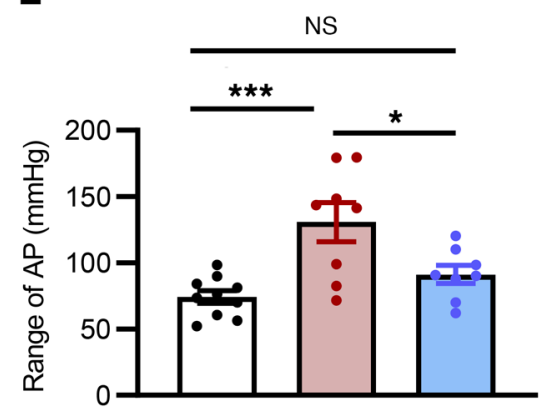

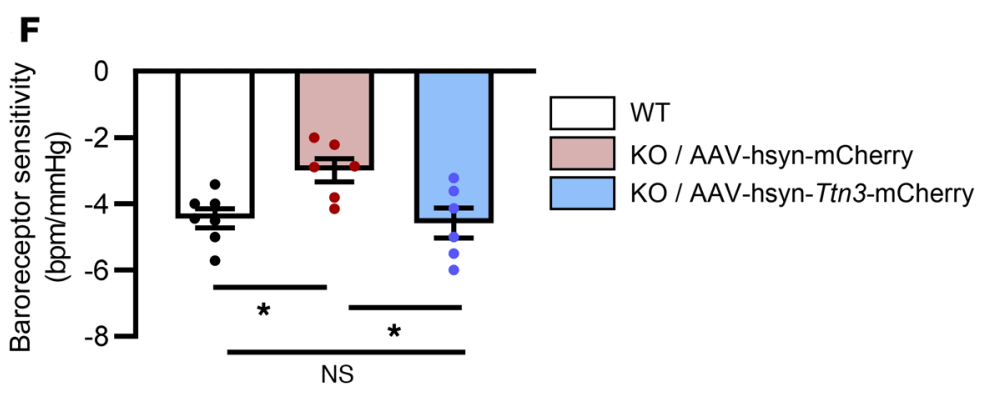

Figure 7. Overexpression of $\operatorname{Ttn} 3$ in NG neurons of $\operatorname{Ttn}^{-1-}$ mice rescues the loss of baroreceptor function in these mice. (A) mCherry expression in NGs of $\mathrm{Ttn}^{-1-}$ mice infected with AAV-hsyn-Ttn3-mCherry. DIC, differential interference contrast. Scale bar: $100 \mu \mathrm{m}$. (B) Infection efficiency (percentage of total cells) in NGs of Ttn ${ }^{-1-}$ mice infected with AAV-hsyn-mCherry (red) or AAV-hsyn-Ttn3-mCherry (blue). Data represent the mean \pm SEM. (C-E) Summaries of MAP (C), HR (D), and range of AP (E) recorded over a 24-hour period in WT $(n=10)$ and Ttn3 ${ }^{-/}$mice infected with either AAV-hsyn-mCherry or AAV-hsynTtn3-mCherry ( $n=8$ per group). Data represent the mean $\pm \mathrm{SEM}$. ${ }^{*} P<0.05$, ${ }^{*} P<0.01$, and ${ }^{* * *} P<0.001$, by 1 -way ANOVA with Tukey's post hoc test. (F) Summary of baroreflex sensitivity recorded over a 24-hour period in WT $(n=7)$ and Ttn ${ }^{-1-}$ mice infected with either AAV-hsyn-mCherry or AAV-hsyn-Ttn3mCherry ( $n=6$ per group). Data represent the mean \pm SEM. ${ }^{*} P<0.05$, by 1 -way ANOVA with Tukey's post hoc test.

redundancy in molecular sensors is required for critical physiological functions so that mutations in one type of channel would not have much of an affect on the system $(11,12,14,15,18,49)$. However, TTN3 and Piezo channels may play a different role in sensing AP changes in baroreceptors. Myelinated or unmyelinated fibers in the ADN show different pressure sensitivity and maximal firing rates (50-52). In addition, the baroreflex gain is higher with sinusoidal pressure changes than with steady-state pressure changes $(51,53)$. Recently, transcriptomes of single NG neurons were analyzed using a single-cell RNA-Seq technique (54). On the basis of the unique gene expression patterns, Ernfors and colleagues classified $18 \mathrm{NG}$ neuronal clusters that are consistent with chemo-, baro-, stretch, and volume sensor functions of vagal afferents. According to a cell cluster database (http://ernforsgroup.shinyapps.io/vagalsensoryneurons), $\operatorname{Ttn} 3$ is expressed in all $18 \mathrm{NG}$ cell clusters, with high expression in NG1-3 and NG12-18, which is in sharp contrast to the expression patterns of Piezo1 and Piezo2 (54). Therefore, the 2 groups of channels may have distinct roles in sensing phasic or tonic AP with different sensitivity to AP.

TTN3 has been cloned as a means to search for a candidate gene conferring SA MA currents in dorsal root ganglion neurons (21). Mechanical strokes on HEK cells transfected with Ttn3 evoke robust currents whose magnitudes follow the depth of the mechanical strokes $(21,25)$. However, TTN3 is not activated by mechanical stimuli when overexpressed in Piezo1-deficient HEK cells. Therefore, its gating mechanism has been questioned (24, 25). Previously, Piezo1-independent mechanosensitivity was suggested (55). Coexpression of Piezo1 and Ttn3 on HEK cells evoke a MA current as if they are acting independently (55). Furthermore, TTN3 appears to have a pore region whose mutation leads to changes in the ion permeability, indicating that TTN3 is an independent channel, not a regulator of Piezo1 (55). In the present study, we found that TN3 was activated by mechanical stimuli in Piezo1-deficient cells treated with jasplakinolide, which strongly suggests that TTN3 mechanosensitivity is Piezo1 independent. 
Piezo1-deficient HEK cells may have weak membrane tension, as Piezo2 knockdown reduces focal adhesion as well as stress fibers that are required for the generation of mechanical tension in the membrane $(26,56,57)$. The displacement threshold for activating TTN3 is higher than that for Piezo1 (21). Therefore, TTN3 may not be activated in Piezo1-deficient HEK cells because of possible low traction force or cell stiffness $(26,56)$.

In conclusion, we showed that TTN3 was expressed in the terminals of $\mathrm{ADN}$ in the aortic arch, sensed intra-aortic pressure changes, and actively mediated the baroreceptor reflex. Because the baroreflex stabilizes APs, many fatal diseases such as hypertension, stroke, and heart failure occur when the sensitivity of the baroreflex is blunted. Thus, understanding the molecular mechanisms underlying baroreception may lead to therapies for untreatable cardiovascular diseases.

\section{Methods}

Cell culture and transfection. HEK293T (ATCC CRL-3216) or HEK-P1KO (a gift from Ardem Patapoutian, Scripps Research Institute, La Jolla, California, USA) cells were incubated in DMEM (catalog 11995065, Gibco, Thermo Fisher Scientific) supplemented with 10\% FBS and $100 \mathrm{U} / \mathrm{mL}$ penicillin/streptomycin at $37^{\circ} \mathrm{C}$ in $5 \% \mathrm{CO}_{2}$ air. For the transfection of pIRES2-Ttn3-AcGFP1, $1 \mu \mathrm{g}$ cDNA was applied to HEK293T or HEK-P1-KO cells with Fugene HD (catalog E2311, Promega) and incubated for 2 to 3 days before use. Cells were plated on poly-L-lysine-coated coverslips in 35-mm culture dishes.

Primary culture of NG neurons. Left NGs were dissected from WT or Ttn3 $^{-/-}$mice after anesthetization with $50 \mu \mathrm{L}$ Zoletil 100 (Virbac) and Rompun (Bayer) cocktail (ratio 3:2). The NGs were collected in the culture solution containing ice-cold DMEM/F-12 solution (catalog 11320-033, Gibco, Thermo Fisher Scientific) and $100 \mathrm{U} / \mathrm{mL}$ of penicillin and streptomycin. NGs were incubated for 30 minutes in the DMEM/F-12 medium containing $1 \mathrm{mg} / \mathrm{mL}$ collagenase IA (catalog C9891, MilliporeSigma), washed 3 times with $\mathrm{Mg}^{2+}$ and $\mathrm{Ca}^{2+}$ free HBSS (catalog 14170112, Gibco, Thermo Fisher Scientific), and digested in $0.125 \mathrm{mg} / \mathrm{mL}$ trypsin (catalog 27250018, Gibco, Thermo Fisher Scientific) for 30 minutes at $37^{\circ} \mathrm{C}$. The trypsinized cells were centrifuged at $100 \times g$ for 10 minutes and then washed 5 times with the DMEM/F-12, gently triturated with $1-\mathrm{mL}$ pipettes and plated onto round glass coverslips (Fisher), which had been previously treated with poly-L-lysine $(0.5 \mathrm{mg} / \mathrm{mL})$ and laminin $(10 \mu \mathrm{g} / \mathrm{mL}$, catalog 23017-015, Gibco, Thermo Fisher Scientific). The coverslips were placed into small Petri dishes $(35 \times 12 \mathrm{~mm})$. Cells were incubated in the culture media supplemented with $50 \mathrm{ng} / \mathrm{mL}$ nerve growth factor (catalog N0513, MilliporeSigma) and $5 \mathrm{ng} / \mathrm{mL}$ glial cell line-derived neurotrophic factor (catalog PHC7044, Life Technologies, Thermo Fisher Scientific) and then placed in a $37^{\circ} \mathrm{C}$ incubator supplied with an atmosphere of $5 \% \mathrm{CO}_{2}$. Electrophysiological experiments were performed after 2 to 3 days of incubation.

Whole-cell current recordings. Whole-cell currents of HEK cells or NG neurons were recorded with an Axopatch 200B Amplifier (Molecular Devices). Whole cells were formed after breaking the plasma membrane under the pipette tips. The resistance of glass pipettes was approximately $3 \mathrm{M} \Omega$. Junctional potentials were canceled to zero. The pipette solution contained $130 \mathrm{mM} \mathrm{CsCl}, 2 \mathrm{mM} \mathrm{MgCl}$, and $10 \mathrm{mM}$ HEPES adjusted to $\mathrm{pH} 7.2$ with $\mathrm{CsOH}$. The bath solution contained $140 \mathrm{mM} \mathrm{NaCl}, 5 \mathrm{mM} \mathrm{KCl}, 2 \mathrm{mM} \mathrm{CaCl}, 2 \mathrm{mM} \mathrm{MgCl}_{2}$, and $10 \mathrm{mM}$
HEPES adjusted to $\mathrm{pH} 7.2$ with $\mathrm{NaOH}$. The osmolarity of all solutions was adjusted to 290-300 mOsm with the application of D-mannitol. The holding potential was set at $-60 \mathrm{mV}$. The output of the amplifier was stored on a computer with pClamp 10.0 software (Molecular Devices). Data were sampled at $5 \mathrm{kHz}$ and filtered at $1 \mathrm{kHz}$ with Digidata 1440 (Molecular Devices). Curve fitting was performed using Clampfit 10.0 (Molecular Devices). Time constants of inactivation of MA currents were fitted to single exponentials.

For mechanical stimulation, step mechanical stimuli were applied to the cell surface with a fire-polished glass probe as described previously $(21,55)$. For the mechanical stimulation of NG cells, the glass probe was moved forward from 2.5 to $6.7 \mu \mathrm{m}$ at $0.84-\mu \mathrm{m}$ increments from the initial position set $1 \mu \mathrm{m}$ away from the surface of the cells. For the mechanical stimulation of HEK cells, step mechanical stimuli of $2.5,4.5$, and $6.5 \mu \mathrm{m}$ were applied. The duration of the mechanical stimuli was set at $600 \mathrm{~ms}$. The fine movement of the glass probe was made with a micromanipulator (NC4, Kleindiek Nanotechnik). Time constants of inactivation of MA currents were fitted to the single exponential function. The recorded current was classified as RA, IA, or SA if its inactivation time constant $(\tau)$ was $<10 \mathrm{~ms}, 10<<30 \mathrm{~ms}$, and $>30$ ms, respectively (21).

Animals. Mice were housed in each cage in a 12-hour light/12-hour dark cycle with free access to food and water. Seven- to 8-week-old male C57BL/6J (WT), Ttn3 ${ }^{-/-}$, and Ttn3 ${ }^{\text {Cre }}$ mice (20-24 g) were used. The generation of $\operatorname{Ttn}^{-/-}$mice was described previously (21).

Generation of Ttn $3^{\text {Cre }}$ mice. Ttn $3^{\text {Cre }}$ mice were generated by homologous recombination (or homology-directed repair [HDR]) using the CRISPR/Cas9 system at the Macrogen Company (Seoul, Korea). Briefly, the homology regions from the BAC clone containing a Tmem150c locus were cloned into a plasmid. The start codon sequences of Tmem150c were targeted for the insertion of the Cre recombinase gene. The SV-40 poly(A) signal sequences were located just after the Cre gene. The plasmid, including 1-kb homology arms, was used as an HDR donor, which was verified by enzymatic digestion and sequencing. To avoid cleavage of the HDR donor by a CRISPR/ Cas9-mediated, site-specific double-stranded break, the HDR donor contains a silent mutation on the selected sequence of the guide RNA. $\mathrm{FVB} / \mathrm{N}$ stud males were mated with superovulated FVB/N mice. Fertilized zygotes were collected from oviducts. Cas9 protein, synthetic single-guide RNA, and the HDR donor were mixed and injected into the pronucleus of fertilized zygotes. The injected zygotes were implanted into oviducts of pseudopregnant CD1 mice. The knockin mouse was backcrossed with mice of the C57BL/6J strain, and 2 of these knockin lines were crossed to generate reporter mice. All $T t n 3^{\text {Cre }}$ mice used in this study were maintained as hybrid FVBC57BL/6J mice. Founder pups and offsprings were genotyped to confirm the presence of the knockin gene by PCR (expected size $1741 \mathrm{bp}$ ).

Immunofluorescence. The immunostaining methods used were similar to those described previously $(17,58)$. Briefly, after anesthetizing mice deeply with isoflurane, the mice were perfused with $4 \%$ PFA in PBS. The left NG and aortic arches were isolated from adult WT, $\mathrm{Ttn3}^{--}$, and AAV-hsyn-Ttn3-mCherry-treated mice. The tissues were fixed with $4 \%$ PFA and frozen in Tissue-Tek OCT. The 10- $\mu$ m-thick cryosections were incubated with blocking buffer (4\% BSA in PBS with Tween-20) for 30 minutes and with polyclonal TTN3 (AbFrontier, 1:700) (21) Piezo2 (NBP1-78624, Novus Biologicals, 1:500), PGP9.5 (ab8189, Abcam, 1:500), or NFH (ab4680, Abcam, 1:500) antiserum overnight 
at $4^{\circ} \mathrm{C}$. After a 15 -minute wash, the samples were incubated with Alexa Fluor 488-conjugated goat anti-rabbit antibody (A11008, Life Technologies, Thermo Fisher Scientific, 1:800), Alexa Fluor 546-conjugated goat anti-mouse antibody (A11003, Invitrogen, Thermo Fisher Scientific, 1:600), or Alexa Fluor 555-conjugated goat anti-chicken antibody (A21437, Life Technologies, Thermo Fisher Scientific, 1:800) at room temperature for 1 hour. The nucleus was stained for 10 minutes with Hoechst 33342 (H3570, Thermo Fisher Scientific, 1:2000). Labeled sections were imaged using an LSM700 confocal microscope (Carl Zeiss).

Tissue H\&E staining. The aorta and heart were isolated from WT and $\mathrm{Ttnn}^{-/-}$mice. The tissues were washed with PBS, fixed in $4 \% \mathrm{PFA}$, and frozen in Tissue-Tek OCT. The 10- $\mu$ m-thick cryosections were stored at room temperature to remove moisture and subsequently stained with Mayers hematoxylin (MilliporeSigma) for 10 minutes. The sections were washed in distilled water for 5 minutes, dipped in $0.5 \%$ eosin 10 times, and then immersed in distilled water to stop eosin streaking and then sequentially in $50 \%, 70 \%, 95 \%$, and $100 \%$ ethanol. The sections were immersed in Xylene several times and mounted on glass coverslips with neutral balsam mounting medium.

RT-PCR. mRNA expression of Gapdh, Ttn3, Piezo1, and Piezo2 in NG was detected with reverse transcription PCR (RT-PCR). Total RNA was extracted from NGs collected from WT mice using a Total RNA Extraction Kit (Easy-spin, Intron, catalog 17221) and reverse transcribed into cDNA using Reverse Transcriptase (GoScript, catalog A5003, Promega). Target genes were amplified by using DreamTaq DNA Polymerases (catalog EP0701, Thermo Fisher Scientific). The following primers were used for PCR: Gapdh forward primer, 5'-AATGGTGAAGGTCGGTGTGA-3', Gapdh reverse primer, 5'-CACACCCATCACAAACATGGG-3'; Ttn3 forward primer, 5'-GGGAAGAAATGCAGCGTGTG-3', Ttn3 reverse primer, 5'-TCAGAGTGCCAAACCCGAAG-3'; Piezo1 forward primer, 5'- TTCTtCGgGttgGagaggtA-3', Piezo1 reverse primer, 5'TGtCACCATGtggttaAgGatG-3'; and Piezo2 forward primer, 5'-GTAATCTGGTGGTGGCCCTC-3', Piezo2 reverse primer, 5'-CACGCGACCATTGACTTTGG-3'.

DiI labeling of aortic BR neurons. Mice were deeply anesthetized with $50 \mu \mathrm{L}$ Zoletil 100 (Virbac) and Rompun (Bayer) cocktail (ratio $3: 2$ ). Mice were ventilated with a small animal ventilator (SAR-1000, CWE). After the sternum was cut to expose the chest, we applied Dil (1,1'-dilinoleyl-3,3,3',3'-tetramethylindocarbocyanine perchlorate, catalog D3899, Invitrogen, Thermo Fisher Scientific) crystals (25 $\mathrm{mg} / \mathrm{mL}, 1 \mu \mathrm{L}$ ) to the adventitia of the aortic arch using a nanoinjector device (Nanoliter 2010, World Precision Instruments). After injection, the area was covered with parafilm and a silicone gel (Kwik-Sil, World Precision Instruments). Mice were allowed to recover for 6 to 7 days so that Dil could efficiently trace to neurons in the NG.

Tissue clearing and staining. AAV DJ-EF1 $\alpha$-DIO-EYFP was injected into the left NG of Ttn $3^{\text {Cre }}$ mice. Four weeks after the injection, the mice were anesthetized deeply with isoflurane and perfused with $4 \%$ PFA. Under a dissection microscope, the aortic arch was isolated. The upper part of the aortic arch was further dissected for clearing and staining. The clearing process was described elsewhere (34). Briefly, the isolated aorta was fixed in 4\% PFA for 1 day and incubated in CUBIC-1 solution containing 10\% Triton X-100 (VWR Life Science), $5 \%$ NNNN-tetrakis (2-HP) ethylenediamine (SigmaAldrich), and $10 \%$ Urea (Junsei) in distilled water at $37^{\circ} \mathrm{C}$ for 7 days for clearing. The samples were washed in PBS solution 3 times for more than 2 hours and incubated in PBS containing 3\% normal donkey serum solution and anti-NFH antibody (ab4680, Abcam, 1:200) at $23^{\circ} \mathrm{C}$ for 3 days. After 1 day of washing in $1 \times$ PBS, the samples were immersed in PBS containing 3\% normal donkey serum and Alexa 594-conjugated anti-chicken antibody (703-585-115, Jackson ImmunoResearch, 1:500 dilution) and Alexa Fluor 647-conjugated antiGFP primary antibody (A-31852, Thermo Fisher Scientific, 1:250 dilution) at $23^{\circ} \mathrm{C}$ for 3 days. Samples were washed in PBS for 2 hours, incubated in PBS with 1:5000 DAPI (62248, Thermo Fisher Scientific) for 2 hours, and immersed in the CUBIC-2 solution overnight for refractive index matching (34). The samples were then mounted onto a slide with the CUBIC-2 solution and imaged with an LSM 880 confocal microscope (Carl Zeiss).

Recording of ADN activity. For the in vitro recording of the ADN, mice were anesthetized with $1.5 \%$ isoflurane. After exposing the aortic arch in the chest, the cardiac end of the aorta, brachiocephalic trunk, and the left common carotid and subclavian arteries were ligated. The aorta with ligated arteries was carefully dissected out from the body and placed into an oxygenated bath solution containing $123 \mathrm{mM} \mathrm{NaCl}$, $3.5 \mathrm{mM} \mathrm{KCl}, 0.7 \mathrm{mM} \mathrm{MgSO}_{4}, 1.7 \mathrm{mM} \mathrm{NaH}_{2} \mathrm{PO}_{4}, 2.0 \mathrm{mM} \mathrm{CaCl}, 5.5$ mM glucose, $9.5 \mathrm{mM}$ mannitol, and $10 \mathrm{mM}$ HEPES (pH 7.40). A small glass tube was inserted into the distal end of the aorta, and the aorta covering the glass tube was tied tightly. The glass tube was connected to a fluid-filled pressure transducer and a syringe for controlling the intraaortic pressure. The output signal of the pressure transducer was amplified and fed into a computer.

The nerve activity of the ADN was recorded using a suction electrode with a $70-\mu \mathrm{m}$-diameter tip filled with the bath solution. Suctions were repeated until neuronal activity was identified. After stabilization of ADN activity, the aortic arch was subjected to pressure steps of 50, 100, and $150 \mathrm{mmHg}$. Electrical signals in the suction electrode were amplified with ISO-80 (World Precision Instruments), filtered at 0.3 and $1 \mathrm{kHz}$ to remove low- and high-frequency interference, digitized at a sampling rate of $10 \mathrm{kHz}$ with Digidata 1322 (Molecular Devices), and stored in a computer. The stored traces were later analyzed using pClamp software, version 10.0 (Molecular Devices). Spikes with a voltage amplitude greater than $1 \mathrm{SD}$ of the noise were counted as action potentials. The noise was obtained with signals with no apparent action potentials at $0 \mathrm{mmHg}$ pressure.

Twenty-four-hour recording of AP and HR. The 24-hour AP recording method was modified from a previous protocol (59). Briefly, WT or $\mathrm{Ttn}^{-/-}$mice were initially anesthetized under $4 \%$ isoflurane followed by $1.5 \%$ isoflurane to maintain the anesthetic condition. A subcutaneous pocket was created at the dorsal right side of the mouse, and the telemetric pressure transmitter (PA-C10, Data Science International) was inserted into this pocket. The right carotid artery was isolated, and a tight knot was made using a suture to block blood flow from the aorta. A small incision was made on the artery, and the catheter was inserted into the vessel through this incision. Another suture was tightened around the artery to secure the catheter, and the catheter was then further advanced toward the transverse aorta. Once the required depth of catheter was reached, all suture knots around the catheter were tightened to secure its position. The skin was closed and sterilized with betadine. After 7 days of recovery, a DSI Dataquest Telemetry System (Data Sciences International) was used to record the AP and HR for 24 hours in conscious, freely moving mice, with a sampling rate of 500 $\mathrm{Hz}$ for 10 seconds measured every 5 minutes. 
Baroreflex response test. Mice were anesthetized with $1.5 \%$ isoflurane, and a heparinized saline-filled (50 Unit/mL) polyethylene tube was inserted into the femoral vein. The other end of the tube was plugged, tunneled subcutaneously, and sutured at the dorsal surface of the neck. After the mice recovered for 3 days, the plugged end of the polyethylene tube was cut and connected to a syringe for a drug injection. A bolus injection of phenylephrine $(4 \mu \mathrm{L} / \mathrm{kg}$, i.p., MilliporeSigma) was made to test the baroreflex sensitivity by checking the changes in AP (60). The baroreflex sensitivity is calculated as $\triangle \mathrm{HR}$ divided by $\triangle \mathrm{SAP}$.

ADN electrical stimulation. The electrical stimulation of the ADN has been described elsewhere (12). Briefly, mice of both genotypes were anesthetized by injection of ketamine $(90 \mu \mathrm{g} / \mathrm{g})$ and xylazine $(9.0 \mu \mathrm{g} / \mathrm{g})$ cocktail. The ADN was stimulated by step increases in electrical stimulation with pulses of $1 \mu \mathrm{A}$ current (2-ms duration) with 10-, 20-, 30-, and 40-Hz frequencies for 20 seconds. The MAP and HR were recorded using the telemetric pressure system.

AAV infection of the NG. Ttn $3^{-/}$mice were anesthetized with $1.5 \%$ isoflurane, and the right and left NGs were surgically exposed. A micropipette containing AAV-hsyn-mCherry or AAV-hsyn-Ttn3mCherry was inserted into the NGs (61). The virus solution (100 nL) was injected using a Nanoliter 2010 (World Precision Instruments). Animals were allowed to recover for 5 weeks before the recording of AP, HR, and baroreflex sensitivity.

Chemogenetic inhibition or activation of $\mathrm{TTN}^{+}$neurons in vivo. Selective inhibition or activation of $\mathrm{TTN}^{+}$neurons in the NG was achieved using the DREADD system. $\operatorname{Ttn} 3^{\mathrm{Cre}}$ mice were anesthetized with $1.5 \%$ isoflurane, and the right and left NGs were surgically exposed. A micropipette containing AAV-hsyn-DIO-mCherry, AAV-hsyn-DIO-hM4DimCherry, or AAV-hsyn-DIO- $h M 3 D q$-mCherry was inserted into the NGs (61). Animals were allowed to recover for 5 weeks before the telemetric recording of AP and HR. Mice were allowed to adapt to the intraperitoneal CNO $(2 \mathrm{mg} / \mathrm{kg}, 10 \mu \mathrm{L})$ injection by injection of saline once a day for 3 days before the $\mathrm{CNO}$ experiments. BP and HR signals were then recorded for 15 minutes (as a baseline) before and 40 minutes after the intraperitoneal CNO injections. Data were normalized to the 15-minute baseline. After the completion of experiments, mice were perfused with 4\% PFA solution. NGs were collected to assess mCherry expression. Mice without mCherry expression were excluded from the analysis.

Statistics. Data in the figures are shown as the mean \pm SEM. To compare multiple means, 1-way ANOVA was used with Tukey's or Dunnett's post hoc test. An unpaired, 2-tailed Student's $t$ test was used to compare 2 means. Analyses with $P$ values of less than 0.05 were considered significantly different.

Study approval. All mouse procedures were approved by the Institute of Laboratory Animal Resources of the Seoul National University and the Korea Institute of Science and Technology.

\section{Author contributions}

HJL performed in vivo, ex vivo, immunostaining, and electrophysiological experiments. TLN performed electrophysiological experiments and RT-PCR and designed the viral vectors. GSH and SP performed patch-clamp experiments in HEK cells. Hyesu Kim and Hyungsup Kim genotyped and maintained the mouse lines. DYK and SYK performed tissue clearing and confocal imaging. PDR and YS helped with in vivo AP recording. MOL supervised animal experiments. UO initiated and designed the study and wrote the manuscript.

\section{Acknowledgments}

We thank Donghee Kim at the Rosalind Franklin University of Medicine and Science and Larry Cohen at Yale University for their comments on the manuscript. This research was supported by the National Research Foundation of Korea (1711104860) and the BK21+ Program of Korea.

Address correspondence to: Uhtaek Oh, Brain Science Institute, Korea Institute of Science and Technology (KIST), Hwarang-ro 14-gil, Seongbuk-gu, Seoul, 02792, Korea. Phone number: 82.958.7031; Email: utoh@kist.re.kr.
1. Victor RG. Carotid baroreflex activation therapy for resistant hypertension. Nat Rev Cardiol. 2015;12(8):451-463.

2. Fernandez G, Lee JA, Liu LC, Gassler JP. The Baroreflex in Hypertension. Curr Hypertens Rep. 2015;17(3):19.

3. Kaufmann H, Norcliffe-Kaufmann L, Palma JA. Baroreflex Dysfunction. N Engl JMed. 2020;382(2):163-178.

4. Kumada M, Terui N, Kuwaki T. Arterial baroreceptor reflex: its central and peripheral neural mechanisms. Prog Neurobiol. 1990;35(5):331-361.

5. Guyton AC, Coleman TG, Granger HJ. Circulation: overall regulation. Annu Rev Physiol. 1972;34:13-46.

6. Donoghue S, Garcia M, Jordan D, Spyer KM. Identification and brain-stem projections of aortic baroreceptor afferent neurones in nodose ganglia of cats and rabbits. J Physiol (Lond). 1982;322:337-352.

7. McDonald DM. Morphology of the rat carotid sinus nerve. I. Course, connections, dimensions and ultrastructure. J Neurocytol. 1983;12(3):345-372.

8. Heesch CM. Reflexes that control cardiovascular function. Am JPhysiol. 1999;277(6 Pt 2):S234-S243.

9. Andersen S, Andersen A, de Man FS, NielsenKudsk JE. Sympathetic nervous system activation and $\beta$-adrenoceptor blockade in right heart failure. Eur J Heart Fail. 2015;17(4):358-366.

10. Lanfranchi PA, Somers VK. Arterial baroreflex function and cardiovascular variability: interactions and implications. Am J Physiol Regul Integr Comp Physiol. 2002;283(4):R815-R826.

11. Lau OC, et al. TRPC5 channels participate in pressure-sensing in aortic baroreceptors. Nat Commun. 2016;7:11947.

12. Lu Y, et al. The ion channel ASIC2 is required for baroreceptor and autonomic control of the circulation. Neuron. 2009;64(6):885-897.

13. Sun H, Li DP, Chen SR, Hittelman WN, Pan HL. Sensing of blood pressure increase by transient receptor potential vanilloid 1 receptors on baroreceptors. J Pharmacol Exp Ther. 2009;331(3):851-859.

14. Drummond HA, Price MP, Welsh MJ, Abboud FM. A molecular component of the arterial baroreceptor mechanotransducer. Neuron. 1998;21(6):1435-1441.
15. Zeng WZ, et al. PIEZOs mediate neuronal sensing of blood pressure and the baroreceptor reflex. Science. 2018;362(6413):464-467.

16. Dhaka A, Viswanath V, Patapoutian A. Trp ion channels and temperature sensation. Annu Rev Neurosci. 2006;29:135-161.

17. Cho H, et al. The calcium-activated chloride channel anoctamin 1 acts as a heat sensor in nociceptive neurons. Nat Neurosci. 2012;15(7):1015-1021.

18. Patapoutian A, Peier AM, Story GM, Viswanath V. ThermoTRP channels and beyond: mechanisms of temperature sensation. Nat Rev Neurosci. 2003;4(7):529-539.

19. Julius D. TRP channels and pain. Annu Rev Cell Dev Biol. 2013;29:355-384.

20. Tominaga M, Caterina MJ. Thermosensation and pain. J Neurobiol. 2004;61(1):3-12.

21. Hong GS, et al. Tentonin 3/TMEM150c confers distinct mechanosensitive currents in dorsal-root ganglion neurons with proprioceptive function. Neuron. 2016;91(1):107-118.

22. BrierleySM, Hughes P, Harrington A, Blackshaw LA. Chapter 24 - Innervation of the gastrointes- 
tinal tract by spinal and vagal afferent nerves. In: Said H, ed. Physiology of the Gastrointestinal Tract, 5th ed. Boston, Massachusetts, USA: Academic Press; 2012:703-731.

23. Carr MJ, Undem BJ. Bronchopulmonary afferent nerves. Respirology. 2003;8(3):291-301.

24. Anderson EO, Schneider ER, Matson JD, Gracheva EO, Bagriantsev SN. TMEM150C/Tentonin3 Is a regulator of mechano-gated ion channels. Cell Rep. 2018;23(3):701-708.

25. Dubin AE, et al. Endogenous Piezo1 can confound mechanically activated channel identification and characterization. Neuron. 2017;94(2):266-270.e3.

26. Pardo-Pastor C, et al. Piezo2 channel regulates RhoA and actin cytoskeleton to promote cell mechanobiological responses. Proc Natl Acad Sci USA. 2018;115(8):1925-1930.

27. Holzinger A, Meindl U. Jasplakinolide, a novel actin targeting peptide, inhibits cell growth and induces actin filament polymerization in the green alga Micrasterias. Cell Motil Cytoskeleton. 1997;38(4):365-372.

28. Bubb MR, Senderowicz AM, Sausville EA, Duncan KL, Korn ED. Jasplakinolide, a cytotoxic natural product, induces actin polymerization and competitively inhibits the binding of phalloidin to F-actin.J Biol Chem. 1994;269(21):14869-14871.

29. Visegrády B, Lorinczy D, Hild G, Somogyi B, Nyitrai M. A simple model for the cooperative stabilisation of actin filaments by phalloidin and jasplakinolide. FEBS Lett. 2005;579(1):6-10.

30. Martino F, Perestrelo AR, Vinarský V, Pagliari S, Forte G. Cellular mechanotransduction: from tension to function. Front Physiol. 2018;9:824.

31. Brown SS, Spudich JA. Mechanism of action of cytochalasin: evidence that it binds to actin filament ends. J Cell Biol. 1981;88(3):487-491.

32. Nixon RA, Shea TB. Dynamics of neuronal intermediate filaments: a developmental perspective. Cell Motil Cytoskeleton. 1992;22(2):81-91.

33. Wilkinson KD, Lee KM, Deshpande S, Duerksen-Hughes P, Boss JM, Pohl J. The neuron-specific protein PGP 9.5 is a ubiquitin carboxyl-terminal hydrolase. Science. 1989;246(4930):670-673.

34. Susaki EA, Tainaka K, Perrin D, Yukinaga H, Kuno A, Ueda HR. Advanced CUBIC protocols for whole-brain and whole-body clearing and imaging. Nat Protoc. 2015;10(11):1709-1727.

35. Susaki EA, et al. Whole-brain imaging with single-cell resolution using chemical cocktails and computational analysis. Cell. 2014;157(3):726-739.

36. Krauhs JM. Structure of rat aortic baroreceptors and their relationship to connective tissue. J Neurocytol. 1979;8(4):401-414.

37. Doan TN, Stephans K, Ramirez AN, Glazebrook PA, Andresen MC, Kunze DL. Differential distribution and function of hyperpolarization-activated channels in sensory neurons and mechanosensitive fibers. J Neurosci. 2004;24(13):3335-3343.

38. Song X, et al. Expression of P2X(2) and P2X(3) receptors in the rat carotid sinus, aortic arch, vena cava, and heart, as well as petrosal and nodose ganglia. Purinergic Signal. 2012;8(1):15-22.

39. Min S, et al. Arterial baroreceptors sense blood pressure through decorated aortic claws. Cell Rep. 2019;29(8):2192-2201.e3.

40. James MA, Potter JF. Orthostatic blood pressure changes and arterial baroreflex sensitivity in elderly subjects. Age Ageing. 1999;28(6):522-530.

41. Cowley AW, Liard JF, Guyton AC. Role of baroreceptor reflex in daily control of arterial blood pressure and other variables in dogs. Circ Res. 1973;32(5):564-576.

42. Voss A, et al. Baroreflex sensitivity, heart rate, and blood pressure variability in normal pregnancy. Am J Hypertens. 2000;13(11):1218-1225.

43. Gomez JL, et al. Chemogenetics revealed: DREADD occupancy and activation via converted clozapine. Science. 2017;357(6350):503-507.

44. Wess J, Nakajima K, Jain S. Novel designer receptors to probe GPCR signaling and physiology. Trends Pharmacol Sci. 2013;34(7):385-392.

45. Martinka P, Fielitz J, Patzak A, Regitz-Zagrosek V, Persson PB, Stauss HM. Mechanisms of blood pressure variability-induced cardiac hypertrophy and dysfunction in mice with impaired baroreflex. Am J Physiol Regul Integr Comp Physiol. 2005;288(3):R767-R776.

46. Faris IB, Iannos J, Jamieson GG, Ludbrook J. The carotid sinus baroreceptor reflex in conscious rabbits. J Physiol (Lond). 1980;298:321-331.

47. Thrasher TN. Baroreceptors, baroreceptor unloading, and the long-term control of blood pressure. Am J Physiol Regul Integr Comp Physiol. 2005;288(4):R819-R827.

48. Coste B, et al. Piezo1 and Piezo2 are essential components of distinct mechanically activated cation channels. Science. 2010;330(6000):55-60.

49. Chapleau MW, Abboud FM. Contrasting effects of static and pulsatile pressure on carotid baroreceptor activity in dogs. Circ Res. 1987;61(5):648-658.

50. Brown AM, Saum WR, Yasui S. Baroreceptor dynamics and their relationship to afferent fiber type and hypertension. Circ Res. 1978;42(5):694-702.

51. Brown AM. Receptors under pressure. An update on baroreceptors. Circ Res. 1980;46(1):1-10.

52. Seagard JL, van Brederode JF, Dean C, Hopp FA, Gallenberg LA, Kampine JP. Firing characteristics of single-fiber carotid sinus baroreceptors. Circ Res. 1990;66(6):1499-1509.

53. Schmidt RM, Kumada M, Sagawa K. Cardiovascular responses to various pulsatile pressures in the carotid sinus. Am J Physiol. 1972;223(1):1-7.

54. Kupari J, Häring M, Agirre E, Castelo-Branco G, Ernfors P. An Atlas of Vagal Sensory Neurons and Their Molecular Specialization. Cell Rep. 2019;27(8):2508-2523.e4.

55. Hong GS, Lee B, Oh U. Evidence for mechanosensitive channel activity of tentonin $3 /$ TMEM150C. Neuron. 2017;94(2):271-273.e2.

56. Hung WC, et al. Confinement sensing and signal optimization via piezo1/PKA and myosin II pathways. Cell Rep. 2016;15(7):1430-1441.

57. Oakes PW, Beckham Y, Stricker J, Gardel ML. Tension is required but not sufficient for focal adhesion maturation without a stress fiber template. JCell Biol. 2012;196(3):363-374.

58. Yang YD, et al. TMEM16A confers receptoractivated calcium-dependent chloride conductance. Nature. 2008;455(7217):1210-1215.

59. Butz GM, Davisson RL. Long-term telemetric measurement of cardiovascular parameters in awake mice: a physiological genomics tool. Physiol Genomics. 2001;5(2):89-97.

60. Braga VA, Burmeister MA, Sharma RV, Davisson RL. Cardiovascular responses to peripheral chemoreflex activation and comparison of different methods to evaluate baroreflex gain in conscious mice using telemetry. Am J Physiol Regul Integr Comp Physiol. 2008;295(4):R1168-R1174.

61. Lerch TF, et al. Structure of AAV-DJ, a retargeted gene therapy vector: cryo-electron microscopy at 4.5 Å resolution. Structure. 2012;20(8):1310-1320 